\title{
The impact of Galactic synchrotron emission on CMB anisotropy measurements
}

\section{Angular power spectrum analysis of total intensity all-sky surveys}

\author{
L. La Porta ${ }^{1}$, C. Burigana ${ }^{2,3}$, W. Reich ${ }^{1}$, and P. Reich ${ }^{1}$ \\ 1 Max-Planck-Institut für Radioastronomie, Auf dem Hügel 69, 53121 Bonn, Germany \\ e-mail: laporta@mpifr-bonn.mpg.de \\ 2 INAF - IASF Bologna, via P. Gobetti 101, 40129 Bologna, Italy \\ 3 Dipartimento di Fisica, Universita' degli Studi di Ferrara, via Saragat 1, 44100 Ferrara, Italy
}

Received 7 August 2007 / Accepted 7 November 2007

ABSTRACT

\begin{abstract}
Context. Galactic foreground emission fluctuations are a limiting factor for precise cosmic microwave background (CMB) anisotropy measurements.

Aims. We intend to improve current estimates of the influence of Galactic synchrotron emission on the analysis of CMB anisotropies. Methods. We perform an angular power spectrum analysis (APS) of all-sky total intensity maps at $408 \mathrm{MHz}$ and $1420 \mathrm{MHz}$, which are dominated by synchrotron emission out of the Galactic plane. We subtract the brighter sources from the maps, which turns out to be essential for the results obtained. We study the APS as a function of Galactic latitude by considering various cuts and as a function of sky position by dividing the sky into patches of $\sim 15^{\circ} \times 15^{\circ}$ in size.

Results. The APS of the Galactic radio diffuse synchrotron emission is best fitted by a power law, $C_{\ell} \sim k \ell^{\alpha}$, with $\alpha \in[-3.0,-2.6]$, where the lower values of $\alpha$ typically correspond to the higher latitudes. Nevertheless, the analysis of the patches reveals that strong local variations exist. A good correlation is found between the APS normalized amplitude, $k_{100}=k \times 100^{\alpha}$, at $408 \mathrm{MHz}$ and $1420 \mathrm{MHz}$. The mean APS for $\ell \in[20,40]$ is used to determine the mean spectral index between $408 \mathrm{MHz}$ and $1420 \mathrm{MHz}, \beta_{(0.408-1.4)} \mathrm{GHz} \in$ $[-3.2,-2.9]\left(C_{\ell}(v) \propto v^{-2 \beta}\right)$, which is then adopted to extrapolate the synchrotron APS results to the microwave range.

Conclusions. We use the $408 \mathrm{MHz}$ and $1420 \mathrm{MHz}$ APS results to predict the Galactic synchrotron emission fluctuations at frequencies above $20 \mathrm{GHz}$. A simple extrapolation to $23 \mathrm{GHz}$ of the synchrotron emission APS found at these radio frequencies does not explain all the power in the WMAP synchrotron component even at middle/high Galactic latitudes. This suggests a significant microwave contribution (of about 50\% of the signal) by other components such as free-free or spinning dust emission. The comparison between the extrapolated synchrotron APS and the CMB APS shows that a mask excluding the regions with $\left|b_{\text {gal }}\right| \lesssim 5^{\circ}$ would reduce the foreground fluctuations to about half of the cosmological ones at $70 \mathrm{GHz}$ even at the lowest multipoles. The main implications of our analysis for the cosmological exploitation of microwave temperature anisotropy maps are discussed.
\end{abstract}

Key words. Galaxy: general - radiation mechanisms: non-thermal - methods: statistical - cosmic microwave background

\section{Introduction}

The possibility to study the primordial phases of our Universe and its properties and evolution through CMB anisotropies relies on our capability to precisely extract the cosmological signal from observations (Tegmark et al. 2000). Maps of the microwave sky include many Galactic and extragalactic astrophysical contributions. A correct recovery of the CMB anisotropy field requires an accurate removal of those foreground signals from the observed maps. Current knowledge of the foreground components permits one to retrieve the bulk of the cosmological information encoded in the CMB anisotropy, and, in particular, its angular power spectrum (APS). Nevertheless, a deeper understanding of the foregrounds is crucial to settle important cosmological issues, which arose from the WMAP ${ }^{1}$ results (Naselsky et al. 2006; Naselsky \& Verkhodanov 2006; Chiang et al. 2007) and could potentially be addressed by the forthcoming PLANCK ${ }^{2}$ mission (Tauber 2004). Moreover, it would enable a precise reconstruction of the individual foreground components and

\footnotetext{
http://lambda.gsfc.nasa.gov/

2 http://www.rssd.esa.int/Planck
}

therefore a complete astrophysical exploitation of the satellite data.

Galactic synchrotron emission is the major source of contamination at frequencies below $50 \mathrm{GHz}$ for intermediate and large angular scales, as recently confirmed by the impressive WMAP results (Bennett et al. 2003; Hinshaw et al. 2007). Synchrotron radiation (Rybicki \& Lightman 1979) arises from cosmic ray electrons gyrating in the magnetic field of our Galaxy. The energy spectrum and the density of the cosmic ray electrons as well as the magnetic field strength vary across the Galaxy, therefore the observed synchrotron emission will depend on the frequency and on the region of the sky. Radio observations at $v \lesssim 5 \mathrm{GHz}$ provide the clearest picture of the Galactic synchrotron morphology, since at these frequencies the diffuse non-thermal radiation clearly dominates over all other emission components outside the Galactic plane. In the past, the $408 \mathrm{MHz}$ all-sky map (Haslam et al. 1982) has extensively been used as a template for the Galactic synchrotron emission in foreground separation attempts (e.g. Bouchet \& Gispert 1999; Bennett et al. 2003). In addition, that map as well as other less suited surveys have been exploited to find an appropriate 
parametrization of the synchrotron emission APS to be $i$ ) extrapolated to the microwave range for estimating the contamination of CMB anisotropies at different angular scales (Giardino et al. 2001; Baccigalupi et al. 2001) and ii) used to set priors in foreground separation applications (Tegmark \& Efstathiou 1996; Bouchet et al. 1999; Bouchet \& Gispert 1999). The outcome of these analyses is that the APS of the synchrotron emission computed over large portions of the sky can be modelled by a power law, i.e. $C_{\ell} \sim k \ell^{\alpha}\left(\ell \sim 180^{\circ} / \theta\right)$, with a spectral index $\alpha \sim[-3,-2.5]$ for $\ell \lesssim 200$, corresponding to angular scales $\theta \gtrsim 1^{\circ}$. Clearly, such a general result is just a first step as it does not describe the complexity of the synchrotron emission APS, whose parameters are expected to change with frequency and with sky direction.

We have carried out a detailed analysis of all-sky radio maps and improved on previous attempts by providing an accurate characterization of the synchrotron emission APS. The results obtained for the new $1.4 \mathrm{GHz}$ polarization all-sky survey (Reich et al., in prep.) will be reported in a forthcoming companion paper. The analysis presented in this paper focuses on the synchrotron emission APS in total intensity. A substantial improvement was possible by using a new all-sky map at $1.42 \mathrm{GHz}$ (Reich et al., in prep.), which has a higher angular resolution and better sensitivity, in addition to the all-sky map at $408 \mathrm{MHz}$. These maps are currently the best suited data for studying the Galactic synchrotron emission at largest angular scales. A more detailed description of some technical aspects related to this work can be found in La Porta (2007). We extensively used the HEALPix ${ }^{3}$ software package (Górski et al. 2005). HEALPix (Hierarchical Equal Area isoLatitude Pixelization) is a curvilinear partition of the sphere optimized for fast spherical harmonics transforms and angular power spectrum estimation. The latter task is performed by the facility Anafast. We also made use of the data reduction package based on the NOD2-software (Haslam 1974).

The paper is organized as follows. Section 2 describes the characteristics of the $408 \mathrm{MHz}$ and $1.42 \mathrm{GHz}$ total intensity surveys, their projection onto HEALPix maps and noise considerations. In Sect. 3 the Galactic radio emission APS over large areas is examined, which reveals the necessity of a discrete source subtraction for a correct evaluation of the diffuse synchrotron APS. The two all-sky maps are decomposed into a map of the diffuse component and a map of discrete sources. Their angular power spectra are derived and discussed. The results obtained by fitting the angular power spectra of the diffuse component maps are presented. In Sect. 4 the radio survey angular power spectra are extrapolated to the microwave range for a comparison with the WMAP 3-yr results. Sect. 5 is dedicated to a local analysis of the radio map APS. We summarize our results and conclusions in Sect. 6.

\section{The data}

The present analysis focuses on the APS of the Galactic synchrotron emission at radio frequencies. However, the $23 \mathrm{GHz}$ synchrotron component obtained by Hinshaw et al. (2007) using the WMAP 3-yr data has also been considered to some extent and will be further discussed in Sect. 4 .

${ }^{3}$ http://healpix.jpl.nasa.gov/

\subsection{The $408 \mathrm{MHz}$ and $1420 \mathrm{MHz}$ surveys}

The 408 MHz map (Haslam et al. 1982) was produced by merging different datasets obtained with large parabolic reflector telescopes (Jodrell Bank 76 m, Effelsberg 100 m and Parkes 64 m telescopes - see Fig. 1 of Haslam et al. 1982), using a similar observing strategy and the same calibration procedure. The final map is characterized by an angular resolution of $\theta_{H P B W} \sim 0.85$ and a $20^{\prime}$ pixel rms-noise of about $670 \mathrm{mK}$. The version used in the present analysis was corrected for a zero level problem concerning the portion of the sky observed from Jodrell Bank (Reich \& Reich 1988).

The total intensity map at $1420 \mathrm{MHz}$ has been obtained by combining a northern and a southern sky survey (Reich et al., in preparation). Both surveys are on an absolute temperature scale and zero level by using low resolution sky horn measurements (Testori et al. 2001). This includes a correction for far-side lobe contamination for single-dish telescopes. Receiving systems were used, which provide total intensities unaffected by linear polarization. The northern sky survey was observed with the Stockert $25-\mathrm{m}$ telescope and extends in declination from $-19^{\circ}$ to $90^{\circ}$ (Reich 1982; Reich \& Reich 1986). The southern sky survey was carried out with the Villa Elisa 30-m telescope in Argentina and covers the latitude range $\delta \in\left[-90^{\circ}\right.$ to $\left.-10^{\circ}\right]$ (Reich et al. 2001). Both have an angular resolution $\theta_{H P B W} \sim 36^{\prime}$ and overlap for latitudes in the range $\left[-19^{\circ},-10^{\circ}\right]$. The resulting map has a $15^{\prime}$ pixel rms-noise of $\sim 17 \mathrm{mK}$. The original maps are provided as equidistant cylindrical (ECP) maps. For the present analysis these maps have been projected into the HEALPix (Górski et al. 2005) pixelization scheme adopted by the WMAP and PLANCK Consortia. For this purpose a simple regridding algorithm has been implemented, which is described in detail by La Porta et al. (2005). The reliability of the projection provided by this simple approach has been verified by successfully performing forward and backward transformations between the two tessellation schemes. The produced HEALPix maps have a pixel size of $\sim 7^{\prime}$ (the number of pixels for an allsky map is $N_{\text {pix }}=12 n_{\text {side }}^{2}$; here we used $n_{\text {side }}=512$ ).

\subsection{Noise estimate}

The authors of the radio maps have estimated the rms-noise directly on the ECP maps by calculating the standard deviation of low emission regions. Going from a Cartesian representation of the sphere to the HEALPIX scheme, the rms-noise per pixel should theoretically decrease toward the polar caps, according to the formula:

$\sigma_{\text {pixel,HEALPix }} \sim \frac{1}{\sqrt{N}} \times \sigma_{\text {pixel,ECP }}$

where $N$ is the number of the ECP pixels corresponding to each HEALPix pixel at a fixed latitude. Such a relation holds under the hypothesis that the noise is Gaussian and uncorrelated among the pixels. Both these assumptions are not necessarily satisfied in the examined surveys, in primis because the pixel size is about half the angular resolution. Furthermore, the rms-noise quoted for the $408 \mathrm{MHz}$ and $1420 \mathrm{MHz}$ surveys quantifies the temperature fluctuations per pixel, which is due not only to the receiver white noise, but also to the contribution of unresolved sources and of residual systematics effects (as "scanning strategy effects"), that could not be fully eliminated in the data reduction procedure. The overall rms-noise in the HEALPix maps is therefore probably higher than the guess determined in this way, thus making this formula suitable for deriving a lower limit. With this 
formula we constructed a map of rms-noise at both frequencies. The rms-noise per pixel decreases for increasing latitude and varies between $\sim 10 \mathrm{mK}$ and $\sim 18.7 \mathrm{mK}$ at $1420 \mathrm{MHz}$ and $\sim 0.5 \mathrm{~K}$ and $\sim 0.7 \mathrm{~K}$ at $408 \mathrm{MHz}$. Due to varying integration times, the rms-noise is not constant over the ECP map, but it is expected to diminish toward the celestial poles. Also taking this effect into account, while applying the above formula, the derived estimate decreases at most by a factor $\sim 2$. This way we obtain an optimistic lower limit for the rms-noise in the HEALPix maps. The corresponding APS is computed as:

$C_{\ell}^{\text {noise }} \sim c^{\text {noise }} \sim 4 \pi \sigma^{2} / N_{\text {pix }}$

where $\sigma$ is the mean value of the rms-noise in the considered area and $N_{\text {pix }}$ is the total number of pixels in the HEALPix map. A generous upper limit for the noise APS is provided instead by the high multipole tail of the APS. This way we have bracketed the noise APS for each considered sky region.

\subsection{Statistically significant multipole range}

The HEALPix maps produced at $408 \mathrm{MHz}$ and $1420 \mathrm{MHz}$ contain precise statistical information only for angular scales that are:

- larger than the beam, i.e. for $\theta \gtrsim \theta_{H P B W}$, therefore the maximum multipole relevant in the APS analysis of these surveys is $\ell_{\max } \sim 180^{\circ} / \theta_{H P B W}\left[{ }^{\circ}\right]$;

- smaller than the maximum angular extent of the considered area, $\theta_{\text {cov }}$, so that the coverage sets the minimum multipole. A safe choice is $\ell_{\min } \sim 5 \times 180^{\circ} / \theta_{\operatorname{cov}}\left[^{\circ}\right]$.

\section{The APS of large areas: analysis for various cuts}

To investigate the dependence of the synchrotron emission APS parameters on latitude, the Galactic plane has been "cut off" from the maps at different latitudes by setting to zero pixels with $\left|b_{\text {gal }}\right| \leq b_{\text {cut }}$, where $b_{\text {cut }}=5^{\circ}, 10^{\circ}, 20^{\circ}, 30^{\circ}, 40^{\circ}, 50^{\circ}, 60^{\circ}$. At the same time, this approach preserves the largest possible coverage, important to keep the widest range of statistically significant multipoles. We also considered "asymmetric cuts", i.e. regions with $b_{\text {gal }} \geq b_{\text {cut }}$ (northern cuts) and $b_{\text {gal }} \leq-b_{\text {cut }}$ (southern cuts), thus taking into account the difference between the two Galactic hemispheres. In fact, the northern hemisphere contains a large and bright feature of the radio sky, i.e. the North Polar Spur (NPS), which is widely believed to be an old supernova remnant in the Solar System vicinity (Salter 1983; Egger \& Aschenbach 1995).

We computed the corresponding APS by using the facility Anafast of the HEALPix package and renormalized it to account for the incomplete sky coverage. The angular power spectra derived for $\left|b_{\text {gal }}\right| \geq b_{\text {cut }}$ with $b_{\text {cut }} \geq 40^{\circ}$ are shown in Fig. 1 , as representative examples. All the recovered angular power spectra flatten towards higher multipoles. Such a behaviour of the APS might be due to noise, systematic effects ("stripes"), discrete sources or might be an intrinsic characteristic of the synchrotron emission fluctuation field. Instrumental white noise can be discarded because its APS should be constant, whereas after the flattening the angular power spectra decrease with $\ell$ as in the presence of beam smoothing. "Stripes" are systematic baseline distortions in the telescope's scanning direction and are mainly due to the limited stability of the receiving system and to the influence of weather conditions. They could also be cancelled from the list of possible causes from the comparison with a destriped version of the $408 \mathrm{MHz}$ map (Platania et al. 2003). The
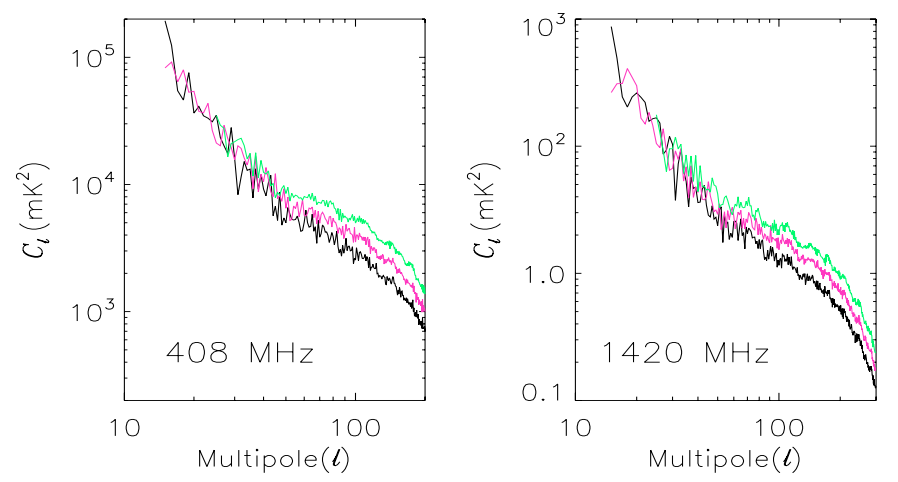

Fig. 1. Angular power spectra of the Galactic plane cut-offs $\left(\left|b_{\text {gal }}\right| \geq\right.$ $\left.b_{\text {cut }}\right)$ at radio frequencies. Each line refers to a certain $b_{\text {cut }}$ (from the bottom: black $\rightarrow 40^{\circ}$, fuchsia $\rightarrow 50^{\circ}$, green $\rightarrow 60^{\circ}$ ).

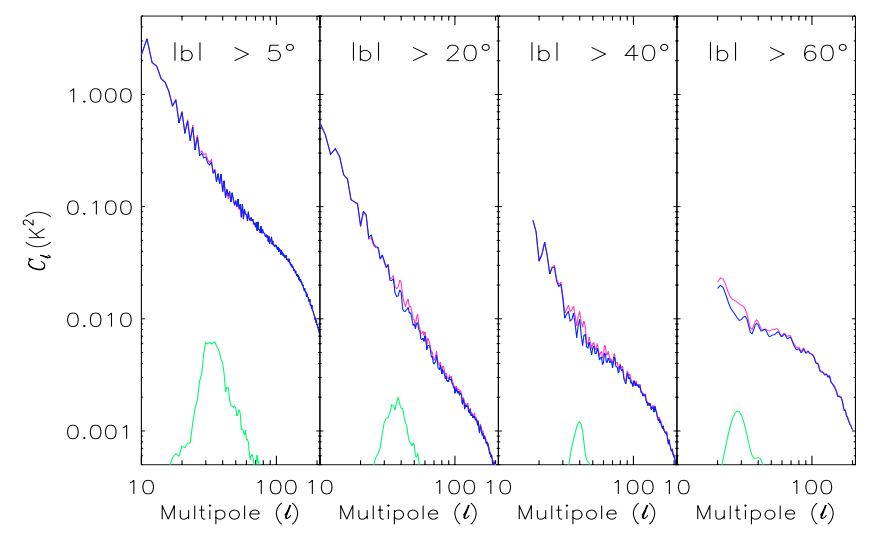

Fig. 2. Comparison between the cut-off angular power spectra of the original (fuchsia lines at the top) and destriped (blue) version of the $408 \mathrm{MHz}$ map. The cut-off angular power spectra of the difference map are also shown (green lines at the bottom).

cut-off APS of the two versions of the $408 \mathrm{MHz}$ map present only marginal differences at intermediate multipoles (see Fig. 2).

\subsection{Discrete source subtraction}

Beside diffuse emission, a large number of discrete sources (DSs) are visible in the radio maps. A DS subtraction has been done by performing a 2-dimensional Gaussian fitting that also provides an estimate of the diffuse background, which is approximated by a tilted plane. Such an estimate has been used to fill the pixels originally corresponding to the subtracted DSs. Where the background emission shows strong gradients the source fitting is more difficult. Consequently, the flux limit above which all discrete sources most likely have been subtracted is different close to the plane and far out of it. Namely, for $|b| \gtrsim 45^{\circ}$ all the sources with peak flux above $\sim 0.8 \mathrm{Jy}$ (respec. $\sim 6.4 \mathrm{Jy}$ ) have been subtracted from the $1420 \mathrm{MHz}$ (respec. $408 \mathrm{MHz}$ ), whereas for $|b| \lesssim 45^{\circ}$ such a source detection threshold is $\sim 4.6 \mathrm{Jy}$ (respec. $\sim 63.8 \mathrm{Jy}$ ). All discrete sources that could be reasonably fitted by a Gaussian profile have been eliminated and two new maps have been generated at $408 \mathrm{MHz}$ and at $1420 \mathrm{MHz}$ (Fig. 3).

The subtracted DSs (see Burigana et al. 2006, for a map) are mostly point sources, except for some rather extended objects, as for instance the radiogalaxy Centaurus A, appearing in the original radio maps right of the Galactic center at $b_{\text {gal }} \sim 20^{\circ}$. Such extended objects are among the brighter subtracted DSs and concentrate along or in the proximity of the Galactic plane. 


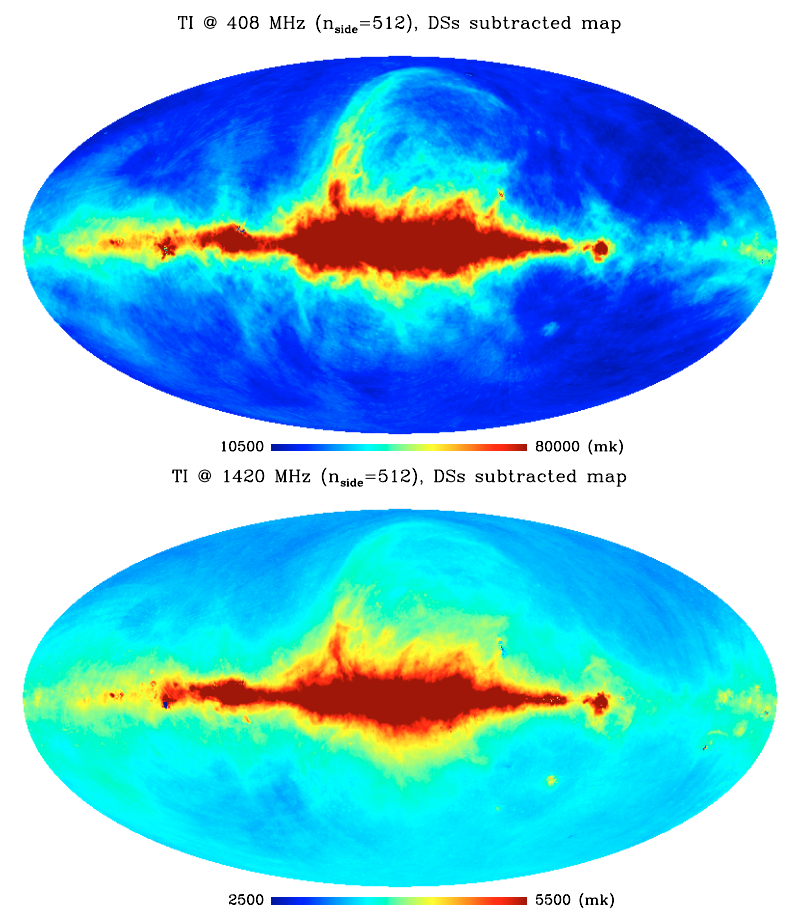

Fig. 3. Mollweide projection of the HEALPix maps produced at $408 \mathrm{MHz}$ (top) and $1420 \mathrm{MHz}$ (bottom) by subtracting the discrete sources. The maps are in Galactic coordinates. The center of the maps is $l=0, b=0$. The Galactic longitude increases toward the left up to $180^{\circ}$.

They are mainly Galactic sources, i.e. HII-regions or supernova remnants. On the contrary, the DSs subtracted at medium and high latitudes are nearly all extragalactic sources.

The maps of some Galactic plane cut-offs at $1420 \mathrm{MHz}$ (respec. $408 \mathrm{MHz}$ ) after DS subtraction are shown in Fig. 4 (respec. Fig. 5). The maps are displayed adopting different scales, in order to emphasize the relative importance of the various components. Note that "scanning strategy effects" are clearly visible in the southern sky at high latitude in the map at $408 \mathrm{MHz}$. As discussed above, the angular power spectra of the destriped and original versions of the Haslam map do not exhibit significant differences, thus implying that "stripes" are not an issue for the APS analysis at $1420 \mathrm{MHz}$. Figure 6 (respec. Fig. 7) shows the APS of the Galactic plane cut-offs at $1420 \mathrm{MHz}$ (respec. $408 \mathrm{MHz}$ ) for the original map, for the DS-subtracted map and for the map of subtracted DSs. The APS of the DS maps almost perfectly matches the flat part of the original map APS at large $\ell$. This result identifies DSs as the reason for the flattening of the original APS and also confirms that the major contribution from source contamination has been eliminated in the DS-subtracted maps. At high latitude the APS of the Galactic fluctuation field is dominated by the DS contribution for $\ell \gtrsim 100$, which is due to the enhanced relative contribution of the DSs respect to the weak diffuse background emission.

Figure 8 shows the APS of the various cuts for the DS map at $1420 \mathrm{MHz}$. Note that for all the southern cuts and for the northern cuts with $b_{\text {cut }} \geq 30^{\circ}$ the DS angular power spectra are rather flat up to $\ell \sim 100$ and then decrease as for a beam smoothing. On the contrary, for the northern cut at $5^{\circ}-20^{\circ}$ the DS angular power spectra present a power law behaviour at lower multipoles, that implies the existence of significant fluctuations also at the larger angular scales, as expected in presence of relatively extended discrete structures. The DS APS of the cut at $20^{\circ}$ is
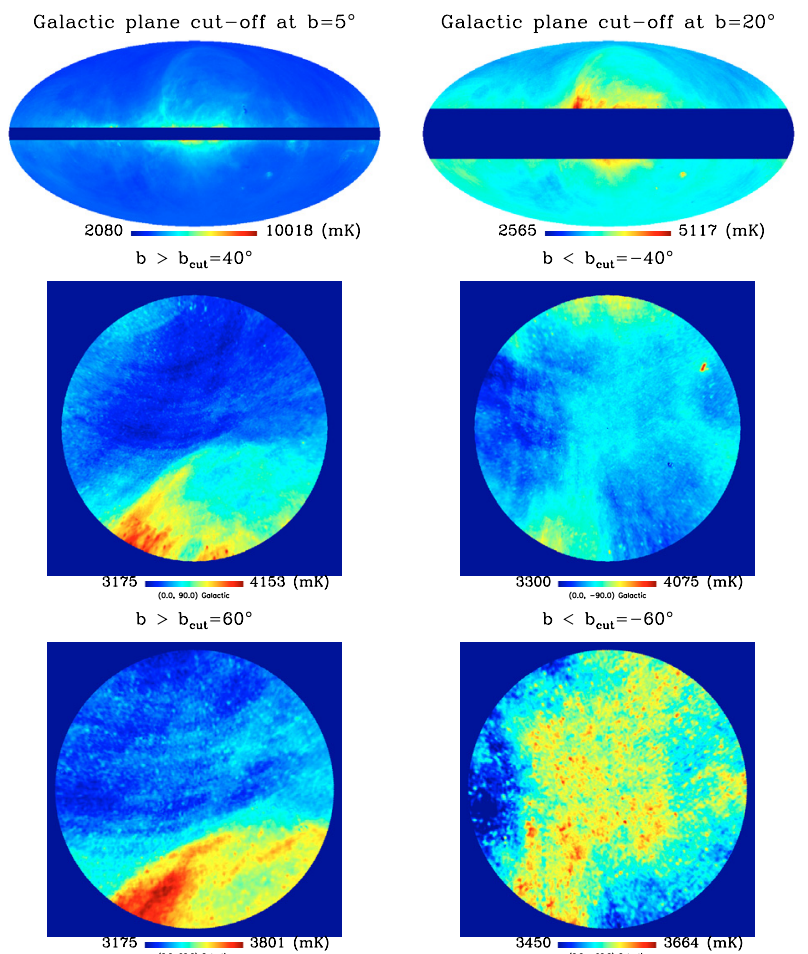

Fig. 4. HEALPix maps of the Galactic plane cut-offs extracted from the $1.4 \mathrm{GHz}$ all-sky map after DS subtraction. A mollweide projection is displayed for the cuts with $\left|b_{\text {cut }}\right| \leq 20^{\circ}$ and a gnomonic view (centered on a Galactic pole) for those with $\left|b_{\text {cut }}\right| \geq 40^{\circ}$.
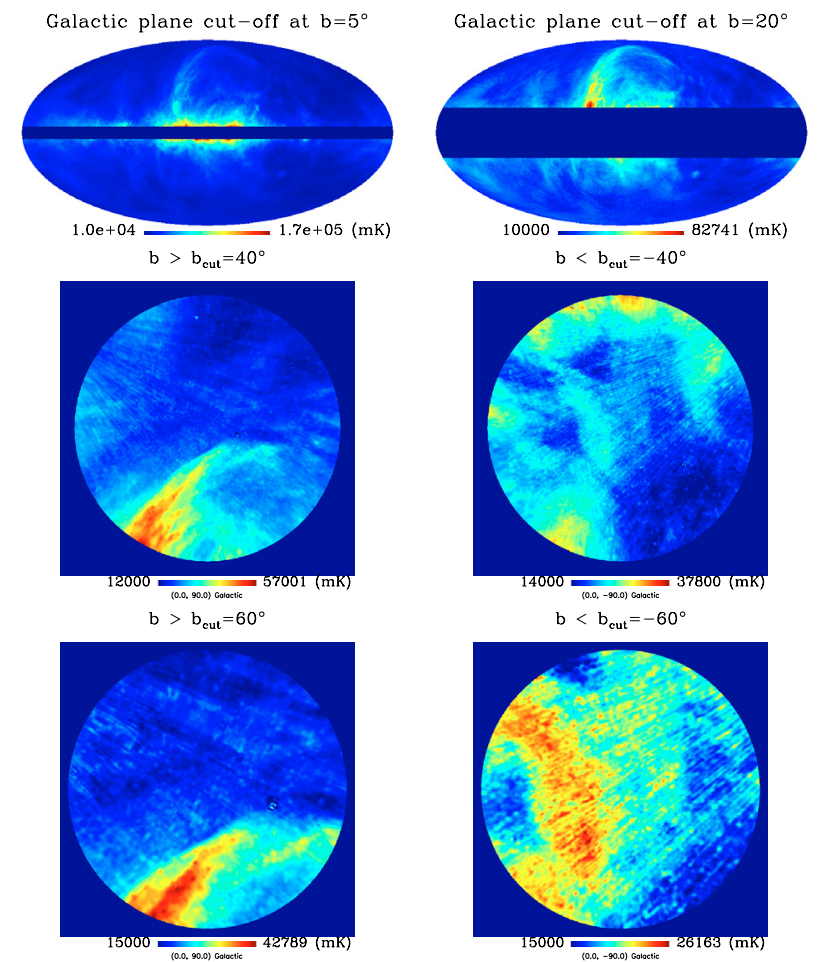

Fig. 5. As in Fig. 4, but at $408 \mathrm{MHz}$.

superimposed on that of the cut at $30^{\circ}$ for $\ell \gtrsim 100$, whereas it exhibits a power law behaviour at lower multipoles. The difference between the angular power spectra of the northern cuts at $20^{\circ}$ and $30^{\circ}$ is then due to the DSs located in the portion of the sky characterized by $20^{\circ} \leq b_{\text {gal }} \leq 30^{\circ}$, which includes Centaurus $\mathrm{A}$, 


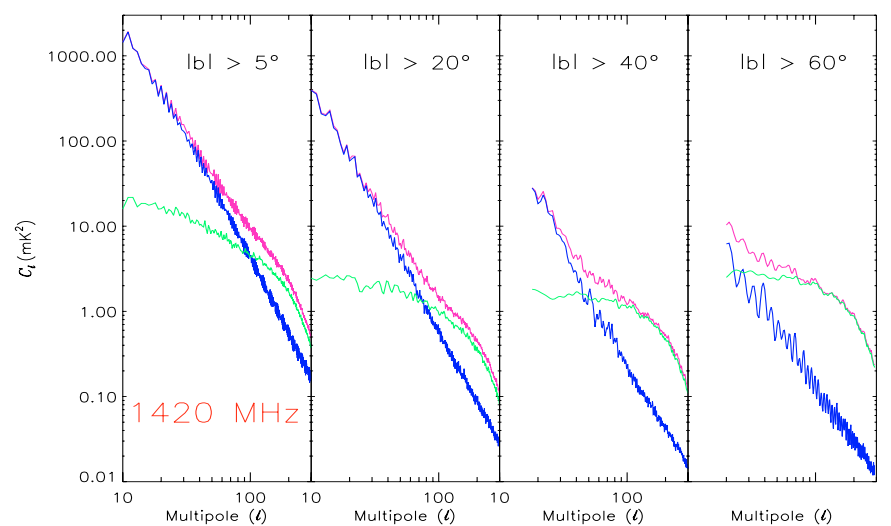

Fig. 6. APS of some Galactic plane cut-off for the $1420 \mathrm{MHz}$ maps: (from the top in each panel) original ( $\rightarrow$ fuchsia), after discrete source subtraction $(\rightarrow$ blue) and DSs only $(\rightarrow$ green $)$.

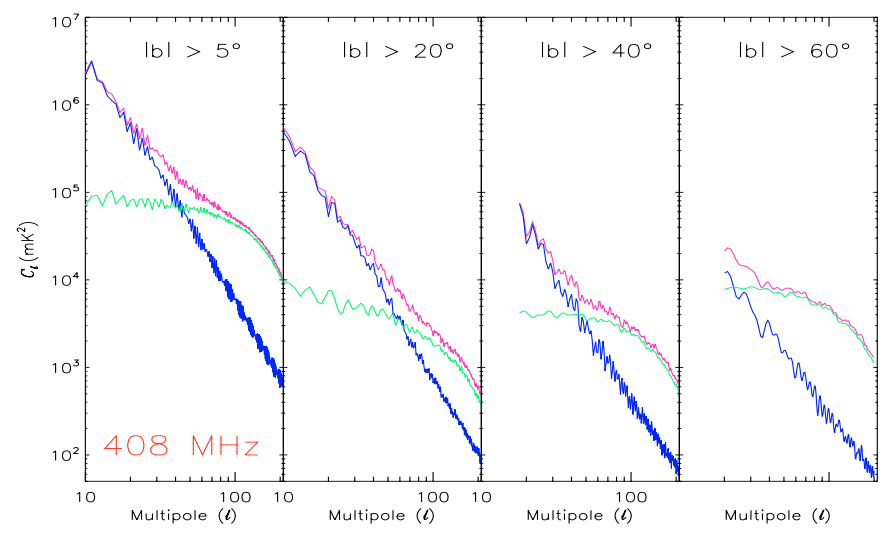

Fig. 7. As in Fig. 6, but at $408 \mathrm{MHz}$.

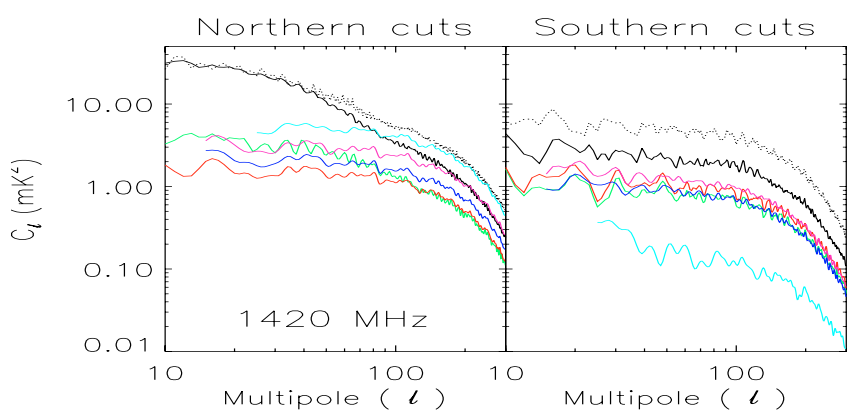

Fig. 8. Angular power spectra of the northern $\left(b_{\text {gal }} \geq b_{\text {cut }} \rightarrow\right.$ left panel $)$ and southern $\left(b_{\text {gal }} \leq-b_{\text {cut }} \rightarrow\right.$ right $)$ cuts for the map of discrete sources at $1420 \mathrm{MHz}$. Color legend (see online version): black (dotted) $\rightarrow\left|b_{\text {cut }}\right|=5^{\circ}$, black $\rightarrow\left|b_{\text {cut }}\right|=10^{\circ}$, green $\rightarrow\left|b_{\text {cut }}\right|=20^{\circ}$, red $\rightarrow\left|b_{\text {cut }}\right|=30^{\circ}$, dark blue $\rightarrow\left|b_{\text {cut }}\right|=40^{\circ}$, fuchsia $\rightarrow\left|b_{\text {cut }}\right|=50^{\circ}$, light blue $\rightarrow\left|b_{\text {cut }}\right|=60^{\circ}$.

thus explaining the power law behaviour of the APS at the lower multipoles. The same situation was found at $408 \mathrm{MHz}$.

\subsection{The APS after source subtraction}

The angular power spectra of the maps after source subtraction approximately follow a power law, as expected for the diffuse Galactic synchrotron emission. For $\left|b_{\text {cut }}\right| \leq 40^{\circ}$ the angular power spectra of all symmetric $\left(|b| \geq b_{\text {cut }}\right)$ and asymmetric ( $b \leq-b_{\text {cut }}, b \geq b_{\text {cut }}$ ) Galactic cuts are very similar to each other and appear progressively shifted downward (see top panels

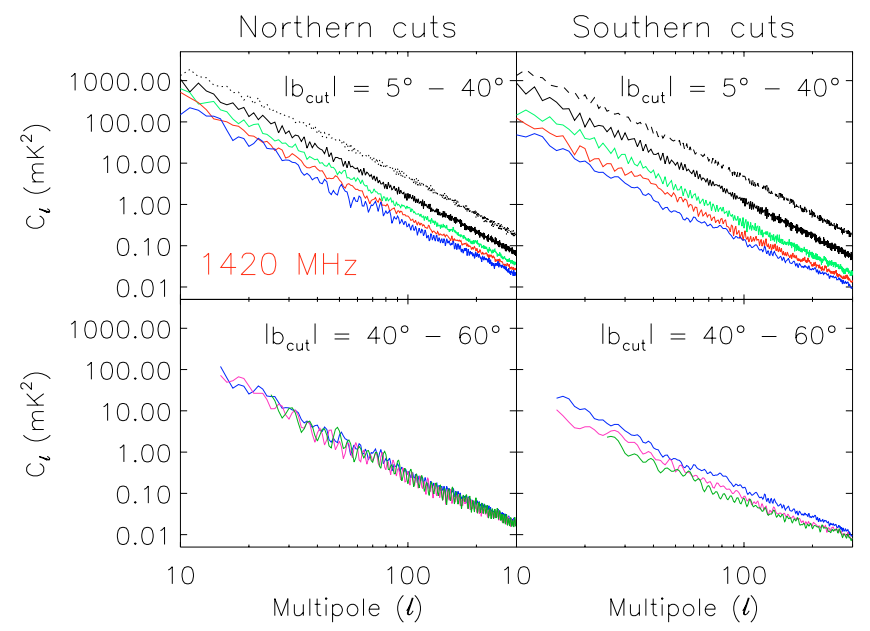

Fig. 9. Comparison between the APS of the cuts for the DS-subtracted map at $1420 \mathrm{MHz}$. The left (respec. right) panels display the angular power spectra of the northern (respec. southern) cuts. First (respec. second) row panels: $b_{\text {cut }}=5^{\circ}-40^{\circ}$ (respec. $b_{\text {cut }}=40^{\circ}-60^{\circ}$ ). Color legend (see online version): black dotted $\rightarrow\left|b_{\text {cut }}\right|=5^{\circ}$, black $\rightarrow\left|b_{\text {cut }}\right|=10^{\circ}$, green $\rightarrow\left|b_{\text {cut }}\right|=20^{\circ}$, red $\rightarrow\left|b_{\text {cut }}\right|=30^{\circ}$, dark blue $\rightarrow\left|b_{\text {cut }}\right|=40^{\circ}$, fuchsia $\rightarrow\left|b_{\text {cut }}\right|=50^{\circ}$, dark green $\rightarrow\left|b_{\text {cut }}\right|=60^{\circ}$.

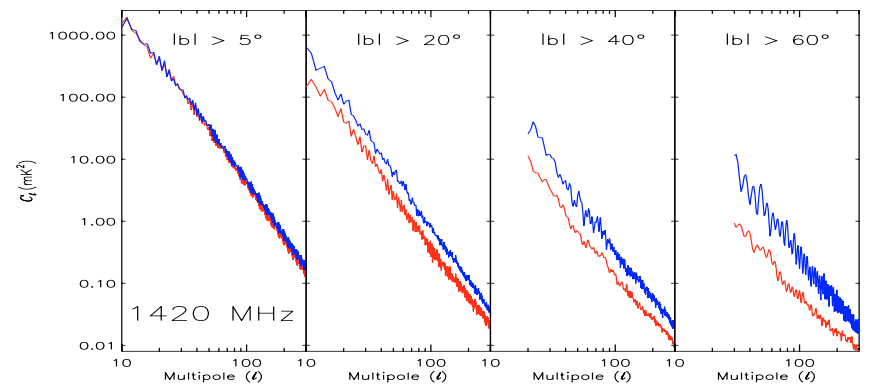

Fig. 10. Comparison between the angular power spectra of the northern $\left(b_{\text {gal }}>b_{\text {cut }}, \rightarrow\right.$ blue - upper lines $)$ and southern $\left(b_{\text {gal }}<-b_{\text {cut }} \rightarrow\right.$ red lower) cuts for the DS-subtracted map at $1420 \mathrm{MHz}$.

of Fig. 9). This result reflects the fact that the Galactic diffuse emission becomes weaker for increasing latitude. The angular power spectra of the symmetric cuts with $\left|b_{\text {cut }}\right| \geq 40^{\circ}$ are superimposed. The same result holds for the angular power spectra of the northern cuts, whereas in the southern hemisphere the APS amplitude decreases for increasing $\left|b_{\text {cut }}\right|$. This discrepancy leads to the conclusion that the angular power spectra of the symmetric cuts with $\left|b_{\text {cut }}\right| \geq 40^{\circ}$ are mainly influenced by the northern hemisphere. Indeed, the angular power spectra of the northern cuts at $b_{\text {cut }} \geq 20^{\circ}$ have amplitudes larger than those of the southern cuts at both frequencies. As an example, Fig. 10 shows the comparison between the angular power spectra of the northern and the southern cuts at $1420 \mathrm{MHz}$. The angular power spectra of the two Galactic hemispheres can reasonably be expected to be similar for the Galactic diffuse synchrotron emission, while they turn out to be different in amplitude and to some extent (mostly at smaller scales) in shape. That difference results from the combination of two effects. In the southern sky, the angular power spectra of the DS-subtracted maps tend to flatten at $\ell \sim 150-200$ due to the presence of unsubtracted sources, whose relative contribution to the fluctuation field increases because of the low background signal. In the northern hemisphere, the Galactic diffuse synchrotron emission is strongly influenced by the radiation of the NPS. 


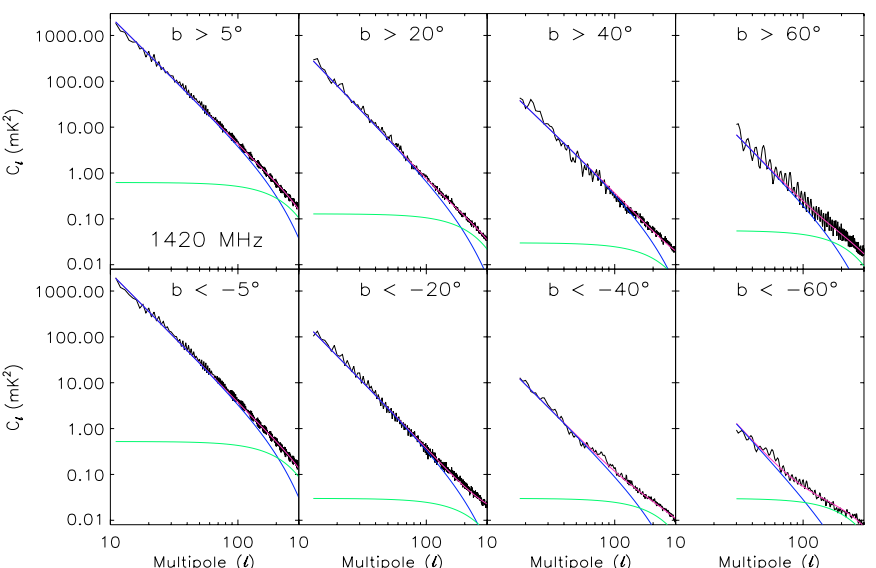

Fig. 11. Angular power spectra of the northern (top panels) and southern cuts (bottom) for the DS-subtracted map at $1420 \mathrm{MHz}$ together with the best fit curves obtained. The individual contributions of synchrotron emission (blue lines) and of sources (green lines) are also plotted (smoothed by the beam).

\subsection{Fit of the APS after source subtraction}

The radio maps after source subtraction include two astrophysical components: the Galactic diffuse emission and the (mainly) extragalactic source contribution, which are convolved with the telescope beam and contaminated by the instrument noise, that can be approximately treated as white noise. We therefore express the corresponding APS as

$C_{\ell}^{\text {map }} \sim\left(C_{\ell}^{\text {synch }}+C_{\ell}^{\mathrm{src}}\right) W_{\ell}+c^{\text {noise }}$

where $W_{\ell}=\mathrm{e}^{-\ell(\ell+1) \sigma_{\mathrm{b}}^{2}}$ is the window function of the symmetric and Gaussian beam ${ }^{4}$, with $\sigma_{\mathrm{b}}=\theta_{H P B W}[\mathrm{rad}] / \sqrt{8 \ln 2}$. The synchrotron emission APS is empirically modelled as $C_{\ell}^{\text {synch }}=$ $k \ell^{\alpha}$. We note that such an empirical choice qualitatively can be explained by magnetohydrodynamic turbulence arguments (Chepurnov 1998; Cho \& Lazarian 2002, 2003). The contribution of the unsubtracted DSs is approximated by a constant term, according to the formalism of Poisson fluctuations from extragalactic point sources (Franceschini et al. 1989). The contribution of fluctuations due to source clustering is expected to be negligible with respect to the Poisson term at the source detection threshold achieved in our maps (Toffolatti et al. 1998).

In order to derive the range of variability of the synchrotron emission amplitude and slope, two extreme cases have been considered (see Appendix C of La Porta 2007 for details). The flattest synchrotron APS compatible with the data is found by neglecting the source term in Eq. (1) and the steepest one is recovered by assuming a null noise contribution and maximizing the source term. We performed a least-square fit to the APS by exploring the parameter space on adaptive grids ${ }^{5}$. The uncertainties on the best fit parameters are derived as the difference with those obtained in the two extreme cases. Figures 11 and 12 show the angular power spectra and the best fit curves corresponding to the best model at the two frequencies, while Tables 1 and 2 list the obtained parameters and their uncertainties.

\footnotetext{
4 The pixel window function has been also taken into account, but its effect is not important here, because the pixel size is significantly smaller than $\sigma_{\mathrm{b}}$.

${ }^{5}$ For this purpose we implemented a specific algorithm and tested its reliability with the MINUITS package of the CERN libraries (James \& Roos 1975).
}

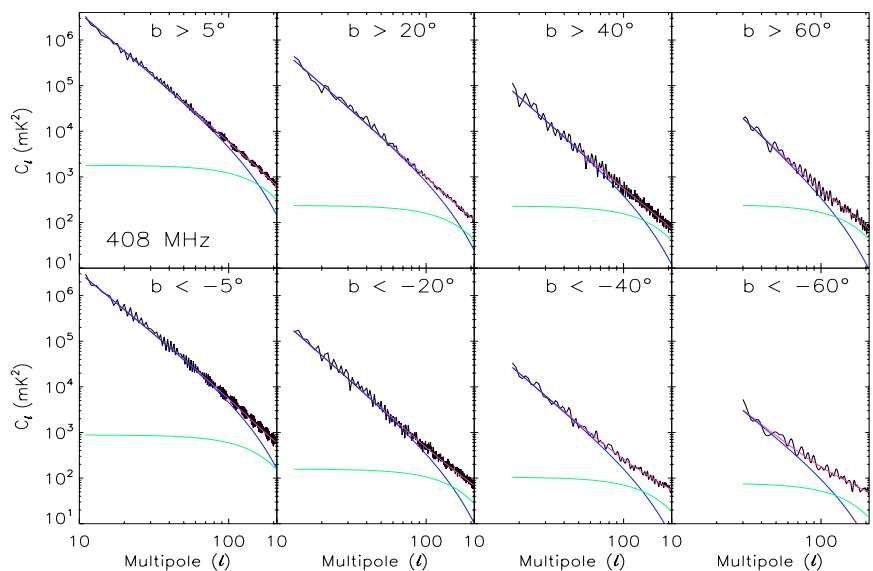

Fig. 12. As in Fig. 11, but at $408 \mathrm{MHz}$.

Table 1. Best fit parameters obtained by modelling the angular power spectra of the northern and southern cuts at $1420 \mathrm{MHz}$ according to Eq. (1).

\begin{tabular}{ccccc}
\hline \hline Coverage & \multicolumn{4}{c}{ Best fit parameters @ 1420 MHz } \\
\hline & $k_{100}\left(\mathrm{mK}^{2}\right)$ & $\alpha$ & $c^{\text {src }}\left(\mathrm{mK}^{2}\right)$ & $c^{\text {noise }}\left(\mathrm{mK}^{2}\right)$ \\
\hline$b_{\text {gal }} \geq 5^{\circ}$ & $4.57_{-0.13}^{+0.95}$ & $-2.75_{-0.03}^{+0.16}$ & $0.621_{+0.079}^{-0.621}$ & $0.0164_{-0.0164}^{+0.1222}$ \\
$b_{\text {gal }} \leq-5^{\circ}$ & $4.00_{-0.09}^{+0.78}$ & $-2.79_{-0.02}^{+0.15}$ & $0.522_{+0.069}^{-0.522}$ & $0.0164_{-0.0164}^{+0.1003}$ \\
$b_{\text {gal }} \geq 10^{\circ}$ & $1.61_{-0.10}^{+0.38}$ & $-2.88_{-0.12}^{+0.21}$ & $0.227_{+0.061}^{-0.227}$ & $0.0151_{-0.0151}^{+0.0425}$ \\
$b_{\text {gal }} \leq-10^{\circ}$ & $1.41_{-0.27}^{+0.13}$ & $-2.74_{-0.28}^{+0.06}$ & $0.128_{+0.107}^{-0.128}$ & $0.0158_{-0.0158}^{+0.0279}$ \\
$b_{\text {gal }} \geq 20^{\circ}$ & $0.78_{-0.07}^{+0.22}$ & $-2.88_{-0.16}^{+0.21}$ & $0.128_{+0.042}^{-0.128}$ & $0.0077_{-0.0077}^{+0.0242}$ \\
$b_{\text {gal }} \leq-20^{\circ}$ & $0.41_{-0.09}^{+0.10}$ & $-2.83_{-0.20}^{+0.13}$ & $0.030_{+0.067}^{-0.030}$ & $0.0146_{-0.0146}^{+0.0047}$ \\
$b_{\text {gal }} \geq 30^{\circ}$ & $0.43_{-0.05}^{+0.17}$ & $-3.02_{-0.02}^{+0.39}$ & $0.128_{+0.004}^{-0.128}$ & $0.0008_{-0.0008}^{+0.0251}$ \\
$b_{\text {gal }} \leq-30^{\circ}$ & $0.22_{-0.06}^{+0.02}$ & $-2.77_{-0.28}^{+0.12}$ & $0.030_{+0.034}^{-0.030}$ & $0.0065_{-0.0065}^{+0.0051}$ \\
$b_{\text {gal }} \geq 40^{\circ}$ & $0.40_{-0.13}^{+0.01}$ & $-2.66_{-0.34}^{+0.25}$ & $0.030_{+0.065}^{-0.030}$ & $0.0113_{-0.0113}^{+0.0071}$ \\
$b_{\text {gal }} \leq-40^{\circ}$ & $0.11_{-0.02}^{+0.05}$ & $-2.77_{-0.34}^{+0.25}$ & $0.030_{+0.022}^{-0.030}$ & $0.0049_{-0.0049}^{+0.0050}$ \\
$b_{\text {gal }} \geq 50^{\circ}$ & $0.21_{-0.02}^{+0.09}$ & $-2.86_{-0.26}^{+0.46}$ & $0.056_{+0.027}^{-0.056}$ & $0.0063_{-0.0063}^{+0.0086}$ \\
$b_{\text {gal }} \leq-50^{\circ}$ & $0.05_{-0.00}^{+0.04}$ & $-3.00_{-0.07}^{+0.63}$ & $0.030_{+0.007}^{-0.030}$ & $0.0026_{-0.0026}^{+0.0066}$ \\
$b_{\text {gal }} \geq 60^{\circ}$ & $0.23_{-0.05}^{+0.06}$ & $-2.81_{-0.33}^{+0.40}$ & $0.056_{+0.030}^{-0.056}$ & $0.0062_{-0.0062}^{+0.0103}$ \\
$b_{\text {gal }} \leq-60^{\circ}$ & $0.03_{-0.00}^{+0.03}$ & $-3.02_{-0.15}^{+0.81}$ & $0.030_{+0.006}^{-0.030}$ & $0.0021_{-0.0021}^{+0.0067}$ \\
\hline & & & &
\end{tabular}

For the synchrotron term, we quote the value of the normalized amplitude $k_{100}=k \times 100^{\alpha}$, which corresponds to a physical quantity. In fact, $k_{100}=C_{\ell=100}$, thus implying that the normalized amplitude gives the mean temperature fluctuations at angular scales of $\sim 2^{\circ}$. Figure 13 shows the best fit parameters of the synchrotron APS as a function of the Galactic latitude. The normalized amplitude, $k_{100}$, is maximum when the considered cut includes the lower latitudes, where the Galactic radio emission peaks. In particular, at $408 \mathrm{MHz}$ (respec. at $1420 \mathrm{MHz}) k_{100} \in[488,6527] \mathrm{mK}^{2}$ (respec. [0.21, 4.57] $\mathrm{mK}^{2}$ ) for the northern cuts and $k_{100} \in[138,6734] \mathrm{mK}^{2}$ (respec. $[0.03,4.00] \mathrm{mK}^{2}$ ) for the southern cuts. The mean error on $k_{100}$ is of $\sim 18 \%$ for the cuts at the lower latitude $\left(\left|b_{\text {cut }}\right| \in\left[5^{\circ}, 30^{\circ}\right]\right)$ and of $\sim 30-40 \%$ for the others. The uncertainty is larger for the cuts at higher latitude due to the reduced multipole range suitable for the fitting procedure. The slope of the synchrotron APS for the northern cuts varies in the interval $\sim[-3.0,-2.8]$ at $408 \mathrm{MHz}$ and $\sim[-3.0,-2.7]$ at $1420 \mathrm{MHz}$, while in the southern 
Table 2. As in Table 1 but at $408 \mathrm{MHz}$.

\begin{tabular}{|c|c|c|c|c|}
\hline \multirow[t]{2}{*}{ Coverage } & \multicolumn{4}{|c|}{ Best fit parameters@ @ $408 \mathrm{MHz}$} \\
\hline & $k_{100}\left(\mathrm{mK}^{2}\right)$ & $\alpha$ & $c^{\mathrm{src}}\left(\mathrm{mK}^{2}\right)$ & $c^{\text {noise }}\left(\mathrm{mK}^{2}\right)$ \\
\hline$b_{\text {gal }} \geq 5^{\circ}$ & $6527 ._{-1349}^{+1306}$ & $-2.78_{-0.22}^{+0.14}$ & $1797 ._{+1223}^{-1797}$ & $187.09_{-187.09}^{+377.84}$ \\
\hline$b_{\mathrm{gal}} \leq-5^{\circ}$ & $6734 ._{-789}^{+743}$ & $-2.68_{-0.11}^{+0.06}$ & $8822_{+1244}^{-882}$ & $291.87_{-291.87}^{+143.62}$ \\
\hline$b_{\mathrm{gal}} \geq 10^{\circ}$ & $2700 ._{-444}^{+467}$ & $-2.80_{-0.21}^{+0.12}$ & $530 ._{+520}^{-530}$ & $95.16_{-95.16}^{+106.22}$ \\
\hline$b_{\text {gal }} \leq-10^{\circ}$ & $1919_{-227}^{+111}$ & $-2.70_{-0.10}^{+0.04}$ & $211_{+438}^{-211}$ & $103.99_{-103.99}^{+53.09}$ \\
\hline$b_{\mathrm{gal}} \geq 20^{\circ}$ & $1147_{-200}^{+215}$ & $-2.83_{-0.19}^{+0.16}$ & 236. & $48.98_{-48.98}^{+39.85}$ \\
\hline$b_{\text {gal }} \leq-20^{\circ}$ & $493 ._{-86}^{+103}$ & $-2.87_{-0.19}^{+0.13}$ & $158 ._{+129}^{-158}$ & $31.71_{-31.71}^{+37.20}$ \\
\hline$b_{\mathrm{gal}} \geq 30^{\circ}$ & $700 ._{-104}^{+135}$ & $-2.91_{-0.16}^{+0.17}$ & $181 .-181$ & $32.95_{-32.95}^{+38.54}$ \\
\hline$b_{\mathrm{gal}} \leq-30^{\circ}$ & $305 ._{-52}^{+112}$ & $-2.88_{-0.14}^{+0.26}$ & $155 ._{+87}^{-155}$ & $21.81_{-21.81}^{+32.50}$ \\
\hline$b_{\mathrm{gal}} \geq 40^{\circ}$ & $572 ._{-111}^{+205}$ & $-2.86_{-0.18}^{+0.34}$ & $230 ._{+106}^{-230}$ & $19.56_{-19.56}^{+41.28}$ \\
\hline$b_{\text {gal }} \leq-40^{\circ}$ & $234 ._{-66}^{+97}$ & $-2.77_{-0.29}^{+0.35}$ & $105 s_{+123}^{-105}$ & $32.47_{-32.47}^{+15.08}$ \\
\hline$b_{\mathrm{gal}} \geq 50^{\circ}$ & $488 ._{-73}^{+246 .}$ & $-3.01_{-0.11}^{+0.46}$ & $208 ._{+117}^{-208}$ & $27.07_{-27.07}^{+23.67}$ \\
\hline$b_{\mathrm{gal}} \leq-50^{\circ}$ & $146 ._{-34}^{+122}$ & $-2.82_{-0.22}^{+0.66}$ & $137 .-137$. & $19.95_{-19.95}^{+22.47}$ \\
\hline$b_{\mathrm{gal}} \geq 60^{\circ}$ & $509 ._{-80}^{+226 .}$ & $-3.04_{-0.26}^{+0.39}$ & 246. & $19.11_{-19.11}^{+45.89}$ \\
\hline$b_{\mathrm{gal}} \leq-60^{\circ}$ & $138 ._{-76}^{+71}$ & $-2.59_{-0.80}^{+0.53}$ & $77 ._{+131}^{-77 .}$ & $32.67_{-32.67}^{+11.66}$ \\
\hline
\end{tabular}

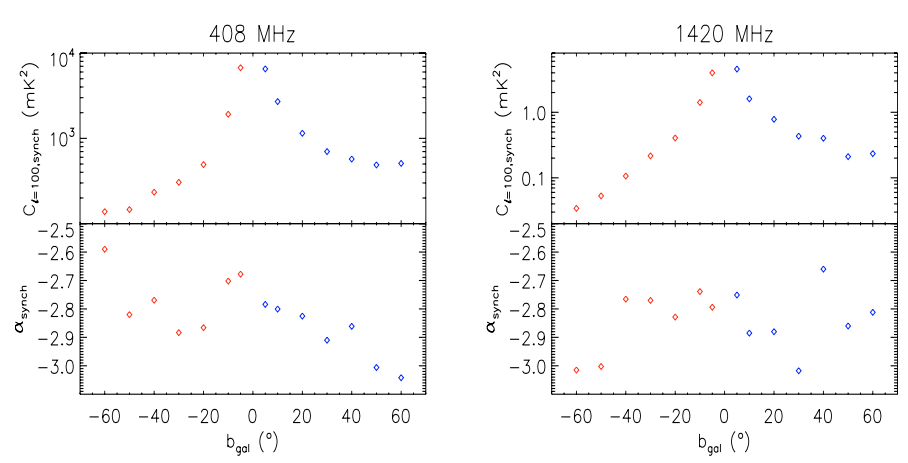

Fig. 13. Best-fit parameters obtained for the Galactic radio synchrotron emission APS against Galactic latitude.

cuts $\alpha \sim[-2.9,-2.6]$ and $\alpha \sim[-3.0,-2.7]$, respectively. The errors on $\alpha$ are on average $\sim(5-7) \%$ for $\left|b_{\text {cut }}\right|<30^{\circ}-40^{\circ}$ and typically increase to $\sim 18 \%$ for cuts at higher latitude.

At both frequencies there is no evidence of a systematic dependence of the synchrotron emission APS slope on latitude (see Fig. 13).

The source term $c^{\text {src }}$ increases for decreasing latitude, as expected given that the source subtraction is less complete below $\sim 45^{\circ}$. From extragalactic source counts at $1.4 \mathrm{GHz}$ in total intensity (Prandoni et al. 2001), the expected source contribution is $c^{\text {src }} \simeq 0.06 \mathrm{mK}^{2}$ for flux densities below $\sim 1 \mathrm{Jy}$ $\left(c^{\text {src }} \simeq[0.03-0.3] \mathrm{mK}^{2}\right.$ including the quoted $1 \sigma$ errors and also considering the effect of the finite sampling) and $c^{\mathrm{src}} \simeq$ $0.30 \mathrm{mK}^{2}\left(c^{\mathrm{src}} \simeq[0.15-1.50] \mathrm{mK}^{2}\right)$ for flux densities below $\sim 5 \mathrm{Jy}$. At $408 \mathrm{MHz}$ the available source counts (Jamrozy 2004) lead to $c^{\text {src }} \simeq 200 \mathrm{mK}^{2}$ for flux densities below $\sim 6.4 \mathrm{Jy}$ $\left(c^{\text {src }} \simeq\left[150-360 \mathrm{mK}^{2}\right)\right.$ and $c^{\text {src }} \simeq 660 \mathrm{mK}^{2}$ for flux densities below $\sim 64 \mathrm{Jy}\left(c^{\mathrm{src}} \simeq[260-1150] \mathrm{mK}^{2}\right)$. The values resulting from the fits are consistent with the above estimates.

\section{Extrapolation to the microwave range}

In this section we extrapolate the results obtained from the analysis of the $408 \mathrm{MHz}$ and $1420 \mathrm{MHz}$ surveys over large areas to the microwave range, in order to make a direct comparison with the WMAP 3-yr results. The main objective of the WMAP mission was the realization of a CMB anisotropy total intensity map and the estimation of the corresponding APS. By-products of the mission are maps of the foregrounds contaminating the cosmological signal at the five frequencies observed by the satellite $(v \sim 23,33,41,61,94 \mathrm{GHz})$, i.e. the microwave emission from the Milky Way, characterized by diffuse (dust, free-free, synchrotron) and discrete (e.g., HII regions, SNRs) components, and from extragalactic sources. The maps were worked out (Hinshaw et al. 2007) by using templates of the various astrophysical components, constructed by exploiting ancillary data. Then a pixel-by-pixel (MEM based) fit of all the maps (see Bennett et al. 2003, for a description of the method), i.e. templates and WMAP frequency maps after subtraction of the $\mathrm{CMB}$ anisotropy field, was performed posing some priors on the spectral behaviour of the foregrounds.

\subsection{Comparison with the WMAP K-band synchrotron component}

The $23 \mathrm{GHz}$ ( $K$-band) synchrotron map by Hinshaw et al. (2007) is considered in this section for a comparison with the $408 \mathrm{MHz}$ and $1420 \mathrm{MHz}$ data. It is evident that such a map provides a picture of the global non-thermal emission observed by the satellite at $23 \mathrm{GHz}$, rather than the Galactic synchrotron component only. Several extragalactic sources are clearly recognizable. Furthermore, as pointed out by Hinshaw et al. (2007), the diffuse non-thermal emission is concentrated at low latitudes and appears remarkably well correlated with the dust component ${ }^{6}$. This might suggest the presence at $23 \mathrm{GHz}$ of anomalous dust emission, as due for example to spinning dust grains (Draine \& Lazarian 1998). In fact, most dusty active star-forming regions are localized along the Galactic plane. This hypothesis seems further supported by the joint analysis of the WMAP maps (1-yr release) and the Green Bank Galactic Plane Survey by Finkbeiner (2004). De Oliveira-Costa et al. (2004) estimated the fluctuations expected at $10 \mathrm{GHz}$ and $15 \mathrm{GHz}$ for the foreground component traced by the K-MEM synchrotron map by Bennett et al. (2003) (1-yr results), by cross-correlating the latter with the Tenerife $10 \mathrm{GHz}$ and $15 \mathrm{GHz}$ CMB maps and all the WMAP CMB maps. They found values one order of magnitude below what is expected for the synchrotron emission and concluded that the K-MEM synchrotron component by Bennett et al. (2003) is dominated by anomalous dust emission even at $\left|b_{\text {gal }}\right| \gtrsim 20^{\circ}$. Hildebrandt et al. (2007) also found evidence for anomalous microwave emission at high Galactic latitudes by cross-correlating the COSMOSOMAS $11 \mathrm{GHz}$ observations with the WMAP $K$ and $K a$-band map. The same conclusion also was reached by Davies et al. (2006), who cross-correlated the WMAP 1-yr map with foreground templates in a dozen small patches located at medium and high latitude. However, the origin of the spatial correlation found at WMAP frequencies between synchrotron and dust emission is still a matter of debate. Bennett et al. (2003) claim that the observed correlation is the result of a spatially varying synchrotron spectral index, which significantly alters the morphology of the synchrotron emission with frequency.

\footnotetext{
6 Such a tight synchrotron-dust correlation holds at all WMAP frequencies.
} 
Hinshaw et al. (2007) affirm that the issue is left open also by the WMAP 3-yr results and that high quality and large coverage surveys at $v \sim 5-15 \mathrm{GHz}$ are needed for a decisive test of both the above discussed explanations of the synchrotron-dust correlation.

The separation of the free-free and synchrotron emission in low latitude regions is also very uncertain. On one hand, the $408 \mathrm{MHz}$ map used as a template of the non-thermal emission contains a non-negligible contribution $(\$ 10 \%)$ of free-free at lower latitudes (Dickinson et al. 2003; Paladini et al. 2005). On the other hand, the $\mathrm{H} \alpha$ map used as template for the freefree emission (Finkbeiner 2003) cannot be properly corrected for dust extinction for $\left|b_{\text {gal }}\right| \lesssim 5^{\circ}$, thus potentially leading to an underestimation of the expected thermal emission at $23 \mathrm{GHz}$. The situation in the vicinity of the Galactic plane is extremely complicated and remains unclear.

The extrapolation of the angular power spectra derived at $408 \mathrm{MHz}$ and $1420 \mathrm{MHz}$ to the microwave range is a delicate issue. The astrophysical components contributing to the fluctuation field APS of the radio surveys scale with frequency in a different way. For the Galactic radio emission between $408 \mathrm{MHz}$ and $1420 \mathrm{MHz}$ Reich et al. (2004) compiled a map of the spectral index $\beta\left(T_{\mathrm{b}} \propto v^{\beta}\right)$ that reveals a complex structure, due to superposition of the spectral behaviour of the map components (synchrotron emission, sources, free-free). The situation is further complicated by a possible but not well known steepening of the diffuse synchrotron emission power spectrum above $10 \mathrm{GHz}$, due to the steepening of the cosmic ray electron energy spectrum (Banday \& Wolfendale 1990, 1991; Strong et al. 2007). Last but not least, the fact that the astrophysical components of the map scale with frequency in a different way may imply a change in the overall APS shape, since the relative weight of the foreground contribution to the APS could vary significantly.

Given the complexity of the open issues discussed above, the following analysis merely aims to verify the consistency between the information about the non-thermal radiation APS coming from the $408 \mathrm{MHz}$ and $1420 \mathrm{MHz}$ data and from the $23 \mathrm{GHz}$ WMAP data. We focused on what happens at medium and high Galactic latitudes, since the problems in interpreting the Galactic emission are more complicated close to the plane.

We first carried out a source subtraction on the $23 \mathrm{GHz}$ map at intermediate and high latitudes $\left(\left|b_{\text {gal }}\right| \gtrsim 40^{\circ}\right)$, similar to that performed on the radio surveys. The comparison of the map of subtracted sources with the mask of sources produced by the WMAP team shows that most $(\sim 80 \%)$ of the objects have been identified and subtracted. We have derived and compared the APS of the original, source-subtracted and source map at $23 \mathrm{GHz}$ for some northern and southern cuts. Namely, we considered $\left|b_{\text {cut }}\right|=40^{\circ}, 50^{\circ}, 60^{\circ}$ and verified that $C_{\ell}^{\text {orig. map }} \sim c^{\text {src }}$ over the significant multipole range (i.e. $\ell \gtrsim 20$ ). This means that in the $23 \mathrm{GHz}$ map the source contribution dominates the high latitude cut APS at all angular scales. In Fig. 14 we show the APS of the $23 \mathrm{GHz}$ map after source subtraction for the asymmetric cuts with $b_{\text {cut }}= \pm 40^{\circ}$. For comparison, we also display the extrapolated radio APS (from Tables 1 and 2), derived as

$C_{\ell}(23)=C_{\ell}\left(v_{\text {radio }}\right) \cdot\left(23 / v_{\text {radio }}\right)^{2 \beta}$

where $v_{\text {radio }}=0.408,1.420 \mathrm{GHz}$. The frequency spectral in$\operatorname{dex} \beta$ is chosen case by case as the value that brings the radio APS to overlay the WMAP one at the lower multipoles (the exact values are reported in the figure caption). The extrapolated angular power spectra are very similar to each other, but steeper than those of WMAP. Furthermore, the frequency spectral indices needed in the extrapolation suggest the existence

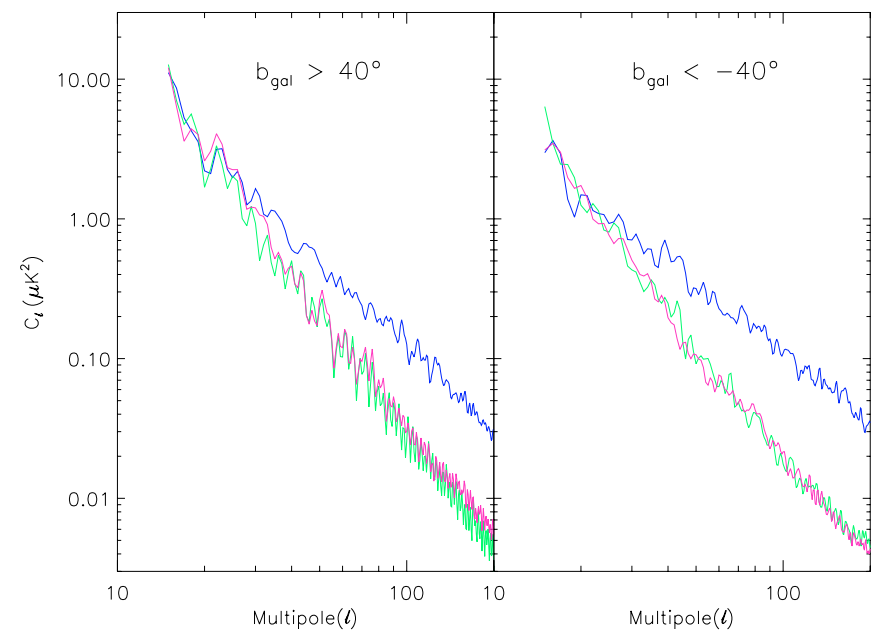

Fig. 14. Angular power spectra of the DS-subtracted radio maps (green $\rightarrow 408 \mathrm{MHz}$, fuchsia $\rightarrow 1420 \mathrm{MHz}$ ) extrapolated to $23 \mathrm{GHz}$ for a direct comparison with those of the WMAP 3-yr DS-subtracted synchrotron component (blue). The radio angular power spectra have been smoothed to $1^{\circ}$ to match the angular resolution of the $23 \mathrm{GHz}$ map. The frequency spectral indices adopted in the extrapolation are $\beta_{(0.408-23) \mathrm{GHz}}=-2.95$ and $\beta_{(1.4-23) \mathrm{GHz}}=-2.90$ for the northern cut and $\beta_{(0.408-23) \mathrm{GHz}}=-2.90$ and $\beta_{(1.4-23) \mathrm{GHz}}=-2.83$ for the southern one.

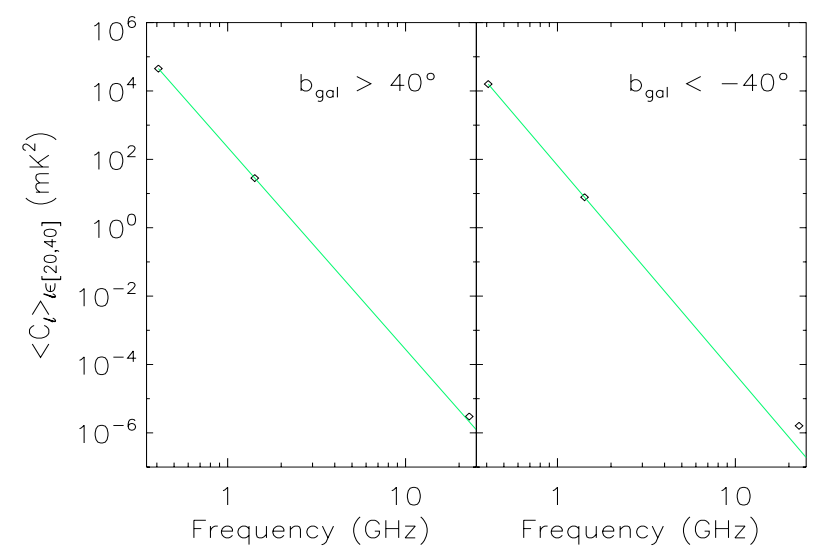

Fig. 15. Mean angular power spectra, $\left\langle C_{\ell}(v)\right\rangle_{\ell \in[20,40]}$, of high latitude cuts $\left(b_{\text {cut }}= \pm 40^{\circ}\right)$ against frequency. The straight line defined by the mean APS of the two lower frequencies is also plotted.

of a steeper spectral behaviour between $408 \mathrm{MHz}$ and $23 \mathrm{GHz}$ than between $1420 \mathrm{MHz}$ and $23 \mathrm{GHz}$. For the Galactic diffuse synchrotron emission we would expect instead $\beta_{(0.408-23) \mathrm{GHz}} \geq$ $\beta_{(1.4-23) \mathrm{GHz}}$ because of the possible steepening of the cosmic ray energy spectrum. In order to obtain a more quantitative estimate of the mean spectral index between the lower frequencies and $23 \mathrm{GHz}$, we used the mean value of the APS of the source-subtracted maps for $\ell \in[20,40]$. In fact, in this range the APS is dominated by the diffuse synchrotron emission, whereas at higher multipoles the APS could still be influenced by the contribution of unsubtracted sources. Figure 15 shows the mean APS as a function of frequency for the cuts with $b_{\text {cut }}= \pm 40^{\circ}$. The spectral index $\beta$ is obtained as

$\left\langle C_{\ell}\left(v_{1}\right)\right\rangle_{\ell \in[20,40]}=\left\langle C_{\ell}\left(v_{2}\right)\right\rangle_{\ell \in[20,40]}\left(v_{1} / v_{2}\right)^{2 \beta}$.

For the northern cut we found $\beta_{(0.408-23) \mathrm{GHz}} \sim-2.92$ and $\beta_{(1.42-23) \mathrm{GHz}} \sim-2.85$. For the southern cuts, $\beta_{(0.408-23) \mathrm{GHz}} \sim$ -2.76 and $\beta_{(1.42-23) \mathrm{GHz}} \sim-2.59$. These values of $\beta$ have a typical uncertainty of a few percent according to the choice of the 


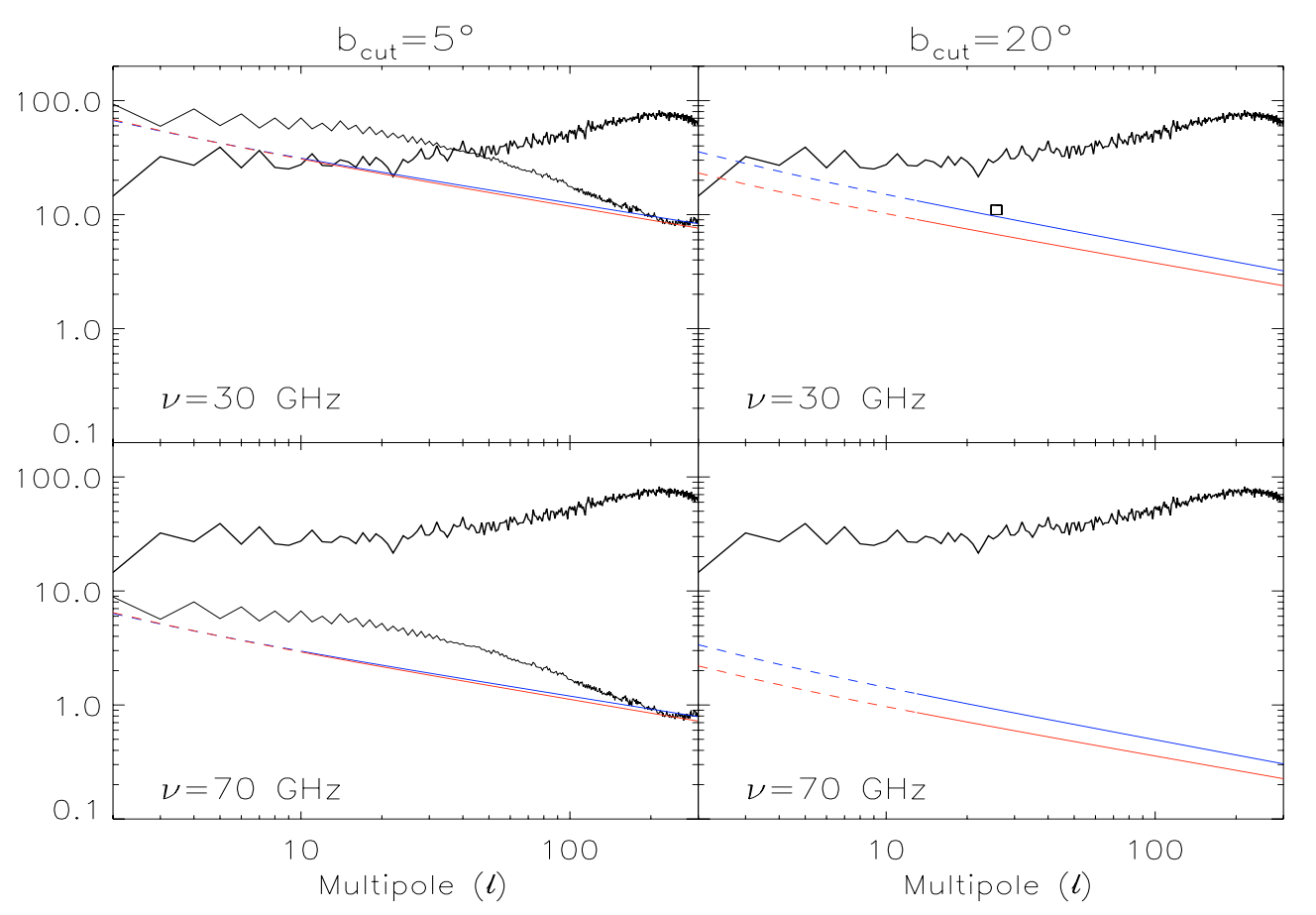

Fig. 16. Comparison between the CMB APS retrieved by Hinshaw et al. (2007) and the synchrotron angular power spectra derived for some cuts of the $1420 \mathrm{MHz}$ map (from Table 1 and Fig. 11), extrapolated to $30 \mathrm{GHz}$ and $70 \mathrm{GHz}$ with a spectral index of -2.9 . Color legend (see online version): blue (upper straight lines) $\rightarrow C_{\ell}^{\mathrm{N}}$ (northern cut), red (lower) $\rightarrow C_{\ell}^{\mathrm{S}}$ (southern cut). The left panels also display the APS for the all-sky (black line) extrapolated as above. The empty square in the top right panel marks the upper limit on synchrotron contamination inferred from COBE-DMR observations (Kogut et al. 1996).

Table 3. Frequency spectral index of the Galactic synchrotron fluctuations between $408 \mathrm{MHz}$ and $1420 \mathrm{MHz}$ derived from the APS.

\begin{tabular}{cccccccc}
\hline \hline \multicolumn{8}{c}{$\beta_{(0.408-1.420) \mathrm{GHz}}$} \\
\hline$\left|b_{\text {cut }}\right|$ & $5^{\circ}$ & $10^{\circ}$ & $20^{\circ}$ & $30^{\circ}$ & $40^{\circ}$ & $50^{\circ}$ & $60^{\circ}$ \\
$b_{\text {gal }}>\left|b_{\text {cut }}\right|$ & -2.9 & -2.9 & -2.9 & -3.0 & -3.1 & -3.2 & -3.2 \\
$b_{\text {gal }}<-\left|b_{\text {cut }}\right|$ & -2.9 & -2.9 & -2.9 & -2.9 & -3.0 & -3.1 & -3.1 \\
\hline
\end{tabular}

multipole range adopted to compute the mean value of the APS. However, it turns out that $\beta_{(0.408-23) \mathrm{GHz}}<\beta_{(1.42-23) \mathrm{GHz}}$ for all reasonable choices of the multipole range. One possible explanation for such a result is that the $23 \mathrm{GHz}$ map includes one or more astrophysical components beside synchrotron emission. On the one hand, the $23 \mathrm{GHz}$ map could still include some Galactic free-free emission, residual from the component separation. Another and likely more relevant candidate is Galactic spinning dust emission. A rough estimate of the excess signal in the $23 \mathrm{GHz}$ map can be obtained by extrapolating the radio results to $23 \mathrm{GHz}$, as shown in Fig. 15. The observed and extrapolated mean APS differ by a factor of $\sim 2.5$ in the northern cut and $\sim 8.4$ in the southern cut. Thus, they differ by factors of $\sim 1.6$ and $\sim 2.9$ respectively in terms of signal in the map. We repeated this calculation by computing the mean APS over other reasonable intervals of multipoles and found in this way that the uncertainty for the given values of the excess signal is about $20 \%$. The difference between the observed and extrapolated values of the mean APS is smaller in the northern hemisphere, which is likely due to the compensatory contribution of the NPS.

Finally, we find that the angular power spectra of the northern and southern sky are almost superimposed at $23 \mathrm{GHz}$ for $\left|b_{\text {cut }}\right| \gtrsim 40^{\circ}$. This can be interpreted as the combination of two effects. The synchrotron emission of the NPS has a steeper frequency spectrum than the average one (Reich \& Reich 1988) and the contribution of emission processes other than synchrotron may be significant at $23 \mathrm{GHz}$. Therefore, the relative importance of the NPS with respect to the overall diffuse background diminishes from $408 \mathrm{MHz}$ and $1420 \mathrm{MHz}$ to microwave frequencies.

\subsection{Synchrotron contamination of the $C M B$ anisotropies}

It is a standard practice to estimate the foreground contamination of CMB anisotropies by means of the corresponding APS, which is usually extrapolated from the frequency range where the foreground component is best observed. For the Galactic synchrotron emission a constant spectral index in the interval $\sim[-2.5,-3.0]$ is commonly adopted, as suggested by the spectral behaviour of the Galactic diffuse emission at radio frequencies. We instead derive the spectral index directly from the results of our APS analysis, thus identifying a proper value for each cut considered. As in the previous section, we compute the mean value of the APS at $408 \mathrm{MHz}$ and at $1420 \mathrm{MHz}$ over the lower multipoles $(\ell \in[20,40])$ and perform a linear extrapolation based on the two points. We prefer to work with the APS of the source-subtracted map rather than with the synchrotron power law derived by fitting it, since the former provides us with a value that exclusively depends on observed data. Our results are reported in Table 3 . The obtained spectral indices vary by a few percent for a different choice of the multipole range used to calculate the mean APS. Figure 16 shows the CMB APS recovered by WMAP (Hinshaw et al. 2007), together with the synchrotron APS derived from the $1420 \mathrm{MHz}$ survey, extrapolated to $30 \mathrm{GHz}$ and $70 \mathrm{GHz}$ (corresponding to the lowest and the highest PLANCK-LFI channels). We display the results obtained for four coverage cases $\left(b_{\text {cut }}= \pm 5^{\circ}, \pm 20^{\circ}\right)$. For comparison, we also extrapolated as above the APS directly extracted from the map for the region at $|b| \geq 5^{\circ}$ and for the all-sky. 
The foreground dominates over the CMB at $30 \mathrm{GHz}$ for a wide multipole range if a mask excluding the Galactic plane is not applied. The frequency spectrum of free-free emission, relevant at low latitudes, is flatter than that of the synchrotron emission. Thus, the extrapolated APS provides a lower limit to the overall Galactic foreground, even neglecting dust emission.

The analysis of the low-frequency maps shows that the APS amplitude of the northern Galactic hemisphere is strongly influenced by the presence of the NPS. Consequently, the results obtained at $1420 \mathrm{MHz}$ for the northern cuts constitute a conservative upper limit for the Galactic diffuse synchrotron emission and can be used together with those of the southern cuts to bracket the synchrotron APS at microwave frequencies.

At $30 \mathrm{GHz}$, a severe contamination is expected from the synchrotron emission up to $\ell \sim 50$ for an almost complete sky coverage $\left(b_{\text {cut }}=5^{\circ}\right)$. A mask excluding the region with $\left|b_{\text {gal }}\right| \leq 20^{\circ}$ reduces the expected synchrotron signal to about half of the CMB anisotropies for $\ell \gtrsim 10$, whereas for lower multipoles the two are comparable. Kogut et al. (1996) examined the COBE-DMR results at $31.5 \mathrm{GHz}$ for $\left|b_{\text {gal }}\right| \gtrsim 20^{\circ}$ and derived an upper limit of $\sim 11 \mu \mathrm{K}$ on the temperature fluctuations due to synchrotron emission on angular scales of $\sim 7^{\circ}$. This value, marked in Fig. 16 by an empty square, is in good agreement with the extrapolated APS for the northern cut at $20^{\circ}$.

At $70 \mathrm{GHz}$, which is the most promising channel for CMB anisotropy measurements since the overall foreground emission reaches a minimum for $v \sim[60,80] \mathrm{GHz}$ (Bennett et al. 2003), the contribution of the Galactic synchrotron emission to the microwave sky fluctuation field is small over the multipole range explored in our analysis $(\ell \gtrsim 10)$. The CMB anisotropies are larger than the foreseen foreground fluctuations by a factor $\gtrsim 10$ for a cut at $5^{\circ}$. For $b_{\text {cut }} \sim 20^{\circ}$ the foreground signal further decreases by a factor $\sim 2$. The extrapolation of our results to $\ell \lesssim 10$ indicates that the cosmological signal should be a factor $\gtrsim 2$ larger than the foreground at the largest angular scales. The precise recovery of the CMB APS for $\ell \lesssim 10$ therefore remains a delicate issue, since the foreground emission is a competitive signal. However, we note that the APS extracted directly from the map shows a certain flattening toward lowest multipoles, slightly improving the situation with respect to the above power law extrapolation.

\section{The APS dependence on sky position: the local analysis}

We have also carried out the analysis of the APS on patches of roughly $14^{\circ} .7 \times 14^{\circ} .7$, in order to describe the local variations of the Galactic emission at $408 \mathrm{MHz}$ and $1420 \mathrm{MHz}$. Significant changes in the amplitude of the synchrotron APS with the considered portion of the sky are expected, since the diffuse radio background gradually increases toward the Galactic plane, where it reaches maximum intensity.

These patches correspond to the pixels of an HEALPix map at $n_{\text {side }}=4$ and allow the study of the angular power spectra on the multipoles range $\sim[60,200-300]$. An angular size $\theta_{\text {patch }} \sim 14.7$ is a good compromise between the wish to divide the sky in a large number of areas and the need to preserve a relatively wide interval of statistically relevant multipoles $\left(\ell \sim 180^{\circ} / \theta\right)$. We have computed the patch angular power spectra for all the versions of the radio maps (original, DS-subtracted and DSs only), both by using the HEALPix facility Anafast and by integrating the two point correlation function (see Appendix D of La Porta 2007, for details). Despite the differences found in

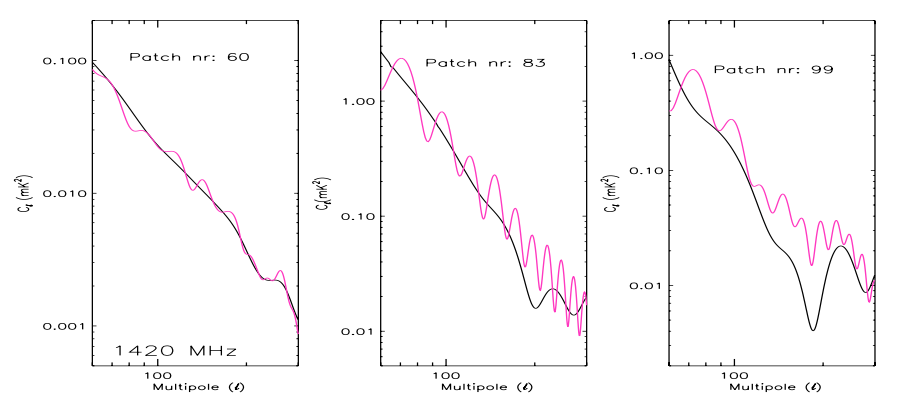

Fig. 17. Comparison between the angular power spectra derived using Anafast (fuchsia) and via integration of the correlation function. Some examples of good (left panel), fair and poor (right) agreement are shown for the map at $1420 \mathrm{MHz}$ after DSs subtraction.

Table 4. Characteristics of the synchrotron APS best fit parameters derived in the local analysis of the $408 \mathrm{MHz}$ map. $k_{100}^{1}$ refers to the patches covering about the brightest half of the sky, which includes the Galactic plane and the NPS. $k_{100}^{2}$ correspond to the other half with weak high latitude emission.

\begin{tabular}{cccccc}
\hline \hline Parameter, $x$ & $x_{\min }$ & $x_{\max }$ & $\langle x\rangle$ & $\sigma_{x}$ & $\%\left(x \in\langle x\rangle \pm \sigma_{x}\right)$ \\
\hline$\alpha$ & -3.50 & -0.70 & -2.70 & 0.60 & 54 \\
$\log \left(k_{100}^{1} / \mathrm{mK}^{2}\right)$ & 2.90 & 5.90 & 3.80 & 0.81 & 70 \\
$\log \left(k_{100}^{2} / \mathrm{mK}^{2}\right)$ & 1.00 & 2.90 & 2.40 & 0.36 & 69 \\
\hline
\end{tabular}

individual cases, on the average there is a good agreement between the angular power spectra derived with the two methods (in Fig. 17 some examples of bad, fair and good cases are shown for the map at $1420 \mathrm{MHz}$ after DS subtraction).

The angular power spectra obtained by using Anafast typically present more oscillations ${ }^{7}$ and tend to be slightly flatter than the correlation function angular power spectra at $\ell \gtrsim 200$. However, the correlation function results are less reliable at higher multipoles, where the choice of the window function might have a non negligible influence. Consequently, we exploited the Anafast angular power spectra in the following analysis.

\subsection{Results}

The patch angular power spectra for the map after DS subtraction are fitted exactly as done in the case of the Galactic cuts (see Sect. 3.3). The maps of the obtained parameters are shown in Figs. 18 and 19. The results derived by using the best model are summarized in Tables 4 and 5. The estimated relative error of the synchrotron APS slope averaged over the ensamble of patches is $|\Delta \alpha / \alpha| \sim 25 \%$ at $408 \mathrm{MHz}$ and $22 \%$ at $1420 \mathrm{MHz}$. The mean relative error of the normalized amplitude, $k_{100}$, is $\sim 25 \%$ at $408 \mathrm{MHz}$ and $20 \%$ at $1420 \mathrm{MHz}$.

The most striking result is that at each frequency the maps of the corresponding parameters, derived by adopting the three different fitting models, show a very similar morphology. Such a resemblance proves that the parameter patterns revealed by the

\footnotetext{
7 Anafast computes the APS in the Fourier space by expressing the temperature fluctuation field in spherical harmonics. The APS is obtained working over the whole sky, even if the map is zero outside the patch taken into account (the derived APS is then renormalized to the case of a full sky coverage). This operation is heuristically equivalent to computing the Fourier transform of a discontinuos function, thus implying a Gibbs effect (Arfken \& Weber 2001).
} 
Fit no SRC

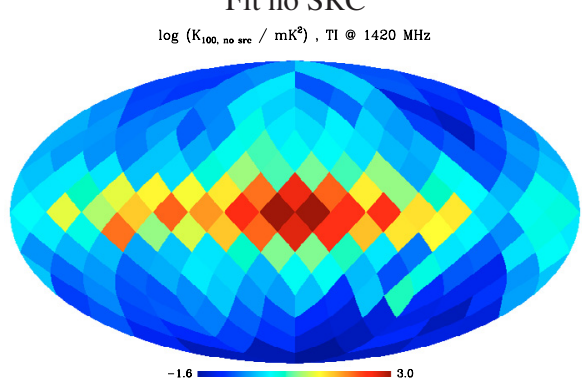

Alpha no sore, Til $1420 \mathrm{MH}$
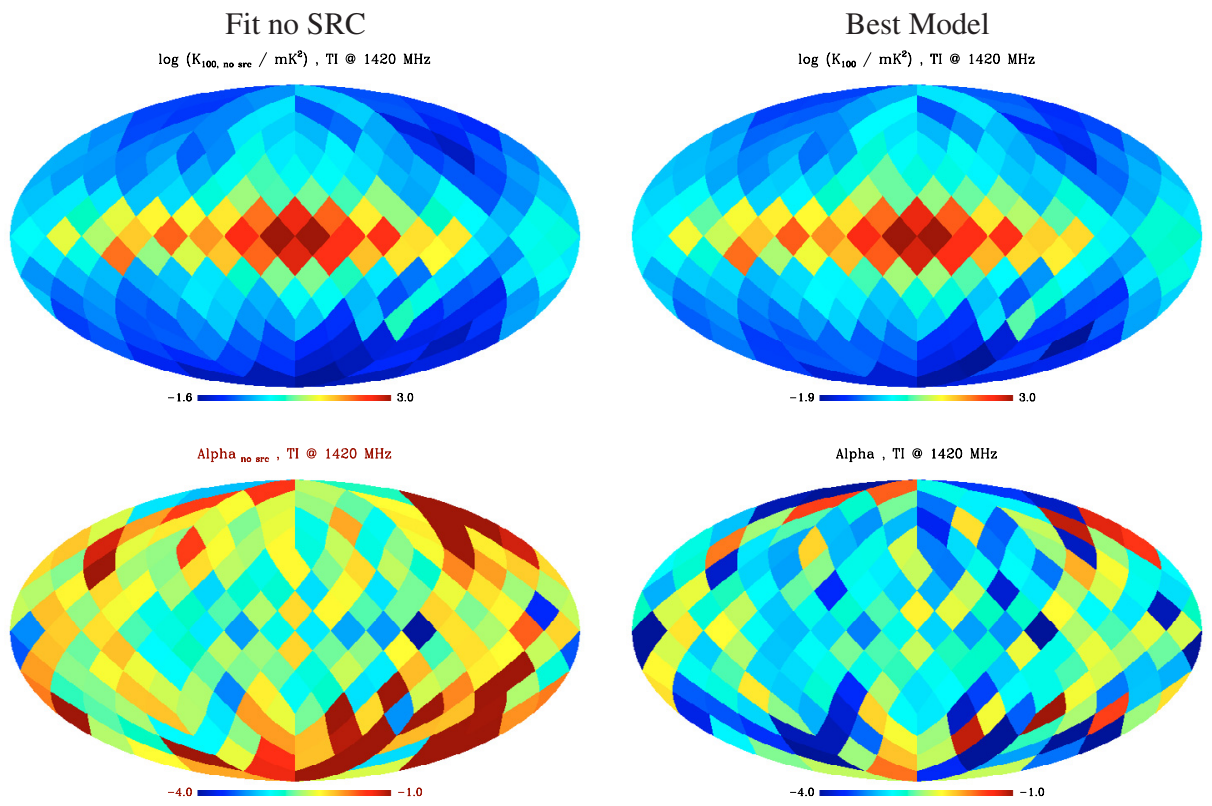

Alpha, TI @ $1420 \mathrm{MHz}$

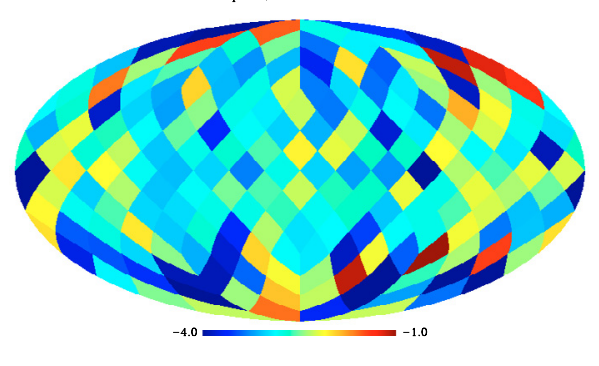

$\log \left(\mathrm{c}^{\text {sec }} / \mathrm{mK}^{2}\right)$, TI $141420 \mathrm{MHz}$

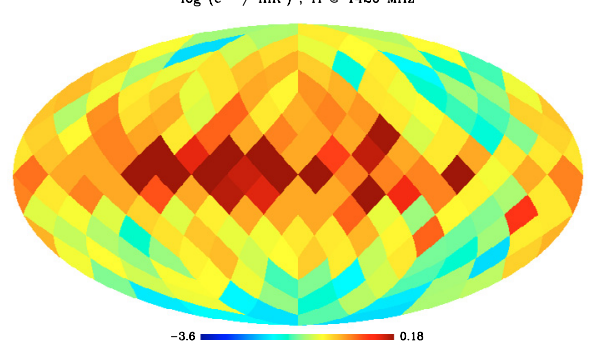

$\log \left(\mathrm{c}^{\text {nolise }} / \mathrm{mK}^{2}\right), T 1 \oplus 1420 \mathrm{MHz}$
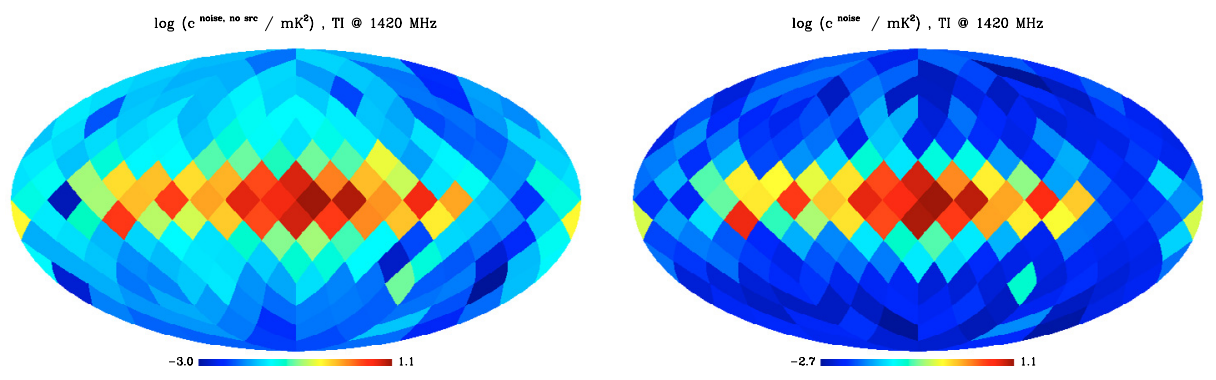

Fit no Noise

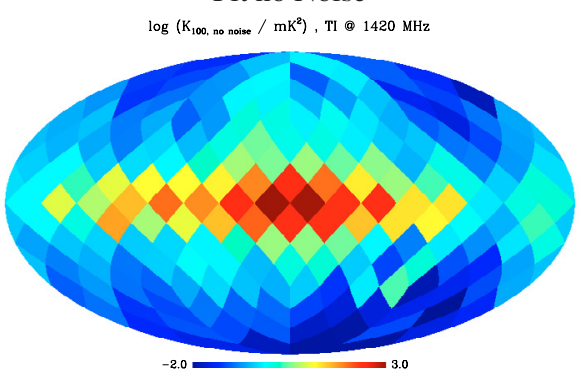

Alpha no osese, , TI @ 1420 MHz

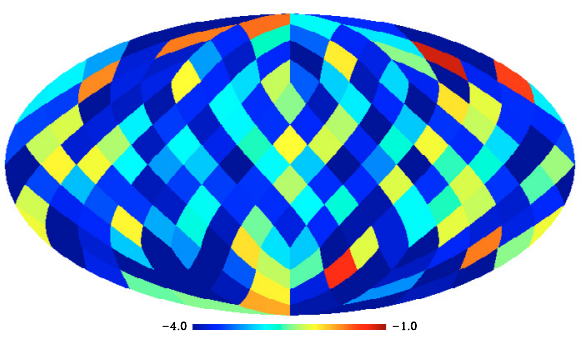

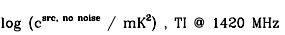

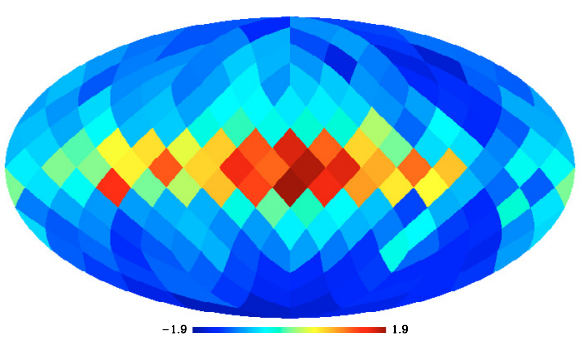

Fig. 18. Maps of the best fit parameters obtained by fitting the angular power spectra of the local analysis patches at $1420 \mathrm{MHz}$. The maps aligned along each row refer to the same parameter. From the top, $\log \left(k_{100}^{\text {synch }} / \mathrm{mK}^{2}\right), \alpha^{\text {synch }}, \log \left(c^{\text {src }} / \mathrm{mK}^{2}\right)$ and $\log \left(c^{\text {noise }} / \mathrm{mK}^{2}\right)$. The first and third columns correspond to the extreme cases, assuming respectively that the source contribution or the noise contamination is negligible.

Table 5. As in Table 4, but at $1420 \mathrm{MHz}$.

\begin{tabular}{cccccc}
\hline \hline Parameter, $x$ & $x_{\min }$ & $x_{\max }$ & $\langle x\rangle$ & $\sigma_{x}$ & $\%\left(x \in\langle x\rangle \sigma_{x}\right)$ \\
\hline$\alpha$ & -4.00 & -1.00 & -2.80 & 0.60 & 70 \\
$\log \left(k_{100}^{1} / \mathrm{mK}^{2}\right)$ & -0.30 & 3.00 & 0.66 & 0.91 & 78 \\
$\log \left(k_{100}^{2} / \mathrm{mK}^{2}\right)$ & -1.90 & -0.31 & -0.85 & 0.38 & 67 \\
\hline
\end{tabular}

local analysis are reliable, despite the uncertainties in the obtained parameter values.

The slope of the synchrotron APS does not show a systematic dependence on Galactic latitude, in agreement with the findings of Sect. 3.3. The normalized amplitude of the synchrotron APS, $k_{100}$, peaks close to the Galactic plane, which reflects the observed morphology. A good correlation is found between the normalized amplitude of the synchrotron APS at $408 \mathrm{MHz}$ and $1420 \mathrm{MHz}$, which is defined by $\log \left(k_{100}^{408} / \mathrm{mK}^{2}\right) \sim A+$ $B \log \left(k_{100}^{1420} / \mathrm{mK}^{2}\right)$, where $A=3.15 \pm 0.02$ and $B=0.88 \pm 0.02$ (see Fig. 20). We note that $10^{A} \sim(408 / 1420)^{2 \beta}$ with $\beta \sim-2.9$, in agreement with the results of Table 3.

The contribution of sources reaches a maximum in the vicinity of the Galactic plane, mainly because a less complete source subtraction was possible for $\left|b_{\text {gal }}\right| \lesssim 45^{\circ}$ than at higher latitudes (see Sect. 3.1). The obtained source terms are in fair agreement with the values estimated by using source counts. This comparison is particularly significant at $1420 \mathrm{MHz}$, where such estimates are more reliable. We summarize the results obtained in this case in Table 6. 

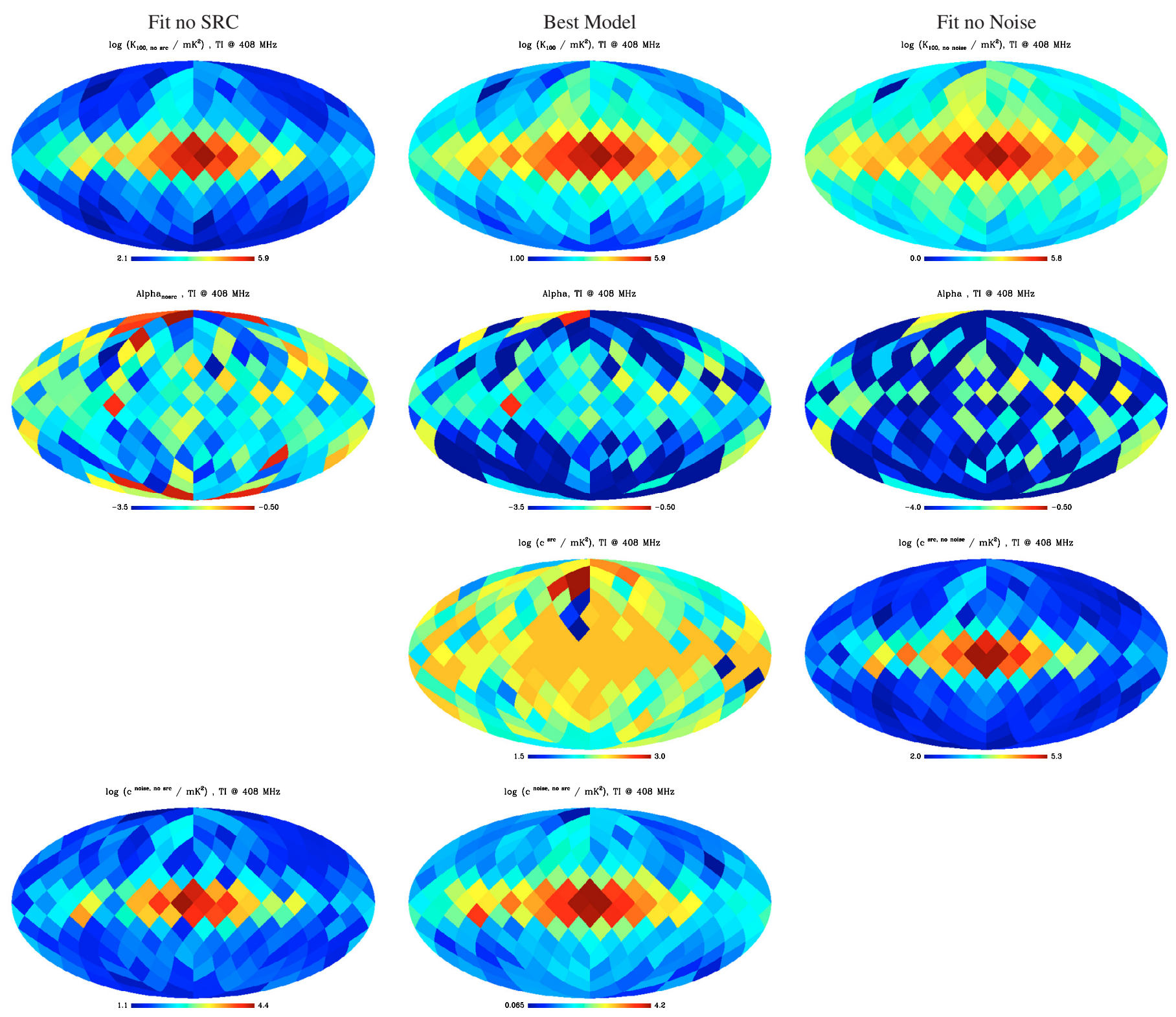

Fig. 19. As in Fig. 18, but at $408 \mathrm{MHz}$.

\section{Summary and conclusions}

The aim of our analysis is to improve our understanding of the Galactic synchrotron emission as a foreground for CMB dedicated experiments. For this purpose, we carried out an unprecedented detailed study of the Galactic radio emission, in terms of its angular power spectrum (APS), using total intensity all-sky maps at $408 \mathrm{MHz}$ and $1420 \mathrm{MHz}$.

An accurate modeling of the synchrotron APS is missing in the literature so far, but is urgently required for a more precise and complete exploitation of the information awaited from the PLANCK satellite. It constitutes a precious input for component separation activities, both for the realization of spatial templates of the foreground and for the definition of priors on its spatial and frequency dependence.

1. Being interested in the diffuse component of the synchrotron emission, the brighter discrete sources (DS) have been eliminated from the radio maps by 2 -dimensional Gaussian fitting.

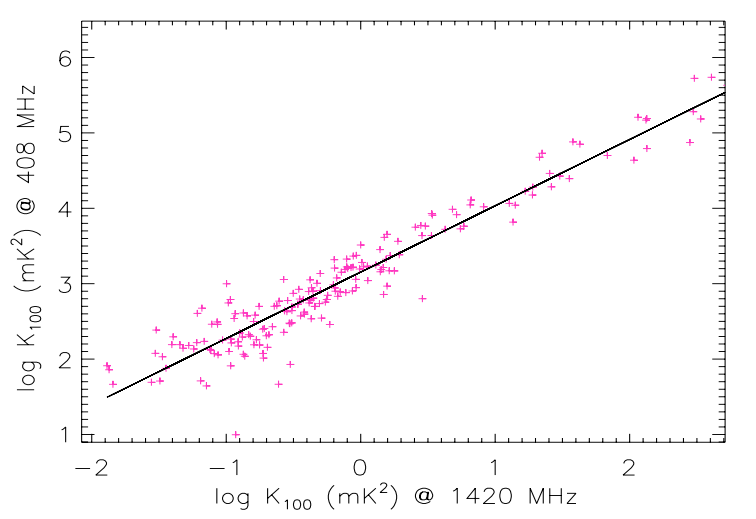

Fig. 20. Correlation between the best fit values obtained for the synchrotron emission APS normalized amplitude $\left(k_{100}\right)$ at $408 \mathrm{MHz}$ and $1420 \mathrm{MHz}$.

This approach is very flexible and also permits the removal of extended structures. 
Table 6. Characteristics of the source term derived in the local analysis at $1420 \mathrm{MHz}$ for $\left|b_{\text {gal }}\right| \gtrsim 45^{\circ}\left(c_{1}^{\text {src }}\right)$ and $\left|b_{\text {gal }}\right| \lesssim 45^{\circ}\left(c_{2}^{\text {src }}\right)$.

\begin{tabular}{cccccc}
\hline \hline Parameter, $x$ & $x_{\min }$ & $x_{\max }$ & $\langle x\rangle$ & $\sigma_{x}$ & $\%\left(x \in\langle x\rangle \pm \sigma_{x}\right)$ \\
\hline $\log \left(c_{1}^{\text {src }} / \mathrm{mK}^{2}\right)$ & -2.30 & -0.75 & -1.50 & 0.42 & 66 \\
$\log \left(c_{2}^{\text {scc }} / \mathrm{mK}^{2}\right)$ & -2.00 & 0.18 & -0.86 & 0.53 & 72 \\
\hline
\end{tabular}

2. The APS was computed for both large areas and small patches and several consistency tests were used to check the reliability of the recovered APS in the case of limited sky coverage (see Appendix B of La Porta 2007).

The study of the APS for various cuts, i.e. of regions with Galactic latitudes above or below a certain value $b_{\text {cut }}\left(\left|b_{\text {cut }}\right| \in\right.$ $\left.\left[5^{\circ}, 60^{\circ}\right]\right)$, allowed us to explore a possible dependence of the mean properties of the Galactic synchrotron emission on latitude, preserving at the same time the largest possible coverage, which is important when estimating the CMB APS because of the sampling variance. Such cuts provided information for $\ell \in\left[\ell_{\min }, \ell_{\max }\right]$, where $10 \lesssim \ell_{\min } \lesssim 30$ for increasing $b_{\text {cut }}$ and $\ell_{\max } \sim 200-300$ at $408 \mathrm{MHz}$ and $1420 \mathrm{MHz}$, respectively.

The patches correspond to the pixels of an HEALPix map at $n_{\text {side }}=4$, which have an angular dimension of $\sim 15^{\circ}$ and permit us to investigate the local variations of the synchrotron APS for multipoles larger than $\sim 60$.

The derived angular power spectra were modelled in both cases according to Eq. (1) and a specific method was set up to find the best least square fit on adaptive grids of the parameter space and to evaluate the uncertanties on the retrieved parameters (see Appendix C of La Porta 2007, for details).

3. An indirect cross-check of the fit result reliability was provided in the case of the Galactic cuts by the estimated source terms, which are consistent with the expectations from extragalactic source counts at both frequencies. Nowadays, source counts at $1.4 \mathrm{GHz}$ are well established down to very low flux limits. It is remarkable that the source angular power spectra obtained independently from source counts and from the fit of the survey APS are in good agreement.

4. The slope of the synchrotron APS, $C_{\ell} \sim k \ell^{\alpha}$, changes with $b_{\text {cut }}$ without showing a well-defined regular trend, although it is found to be typically steeper for $b_{\text {gal }} \gtrsim 20^{\circ}$. For the cuts, $\alpha$ varies in the range $\sim[-3.0,-2.6]$ at both frequencies. However, the analysis of the small patches gives evidence that locally the synchrotron APS can be much flatter for $\ell \gtrsim 60$, reaching in some cases values of $\alpha \sim-0.8$. The normalized amplitude, $k_{100}=k \times 100^{\alpha}$, gradually increases toward the Galactic plane, following, as expected, the background radio emission gradient. A good correlation exists between the results obtained for $k_{100}$ at $408 \mathrm{MHz}$ and $1420 \mathrm{MHz}$, for both cuts and patches. This is expected, given that the spectral properties of the electron density distribution responsible for the Galactic diffuse non-thermal emission should be the same in that frequency range and further supports the reliability of the obtained estimates of the synchrotron APS.

5. The maps of $k_{100}$ and $\alpha$ resulting from the local analysis represent the starting point for the simulation of small-scale fluctuation fields to be added to the DS-subtracted maps to build phenomenological templates of the Galactic synchrotron emission. At present, an empirical approach is the most reliable way to proceed in the realization of realistic templates of the foreground, given the poor knowledge of the Galactic magnetic field and of the cosmic ray electron density distribution needed for a 3-dimensional physical modelling.

The issues (discussed in Sect. 4.1) related to the $K$-band synchrotron component retrieved by Hinshaw et al. (2007) are a clear example of the difficulties encountered in the foreground separation due to the lack of adequate priors and guess. We performed a source subtraction on that map and produced a map of the Galactic diffuse non-thermal emission at $23 \mathrm{GHz}$ to be compared with those at lower frequencies.

6. The extrapolation to $23 \mathrm{GHz}$ of the APS obtained at $408 \mathrm{MHz}$ and $1420 \mathrm{MHz}$ for higher latitude regions $\left(\left|b_{\text {gal }}\right| \gtrsim\right.$ $\left.40^{\circ}\right)$ reveals that the mean spectral index $\left(C_{\ell}(v) \propto v^{2 \beta}\right)$ $\beta_{(0.408-23) \mathrm{GHz}} \lesssim \beta_{(1.4-23) \mathrm{GHz}}$, which is the opposite of what is expected for synchrotron emission. We estimate the excess of the signal in the $23 \mathrm{GHz}$ map to be $\gtrsim 50 \%$ by using the mean value of the APS at lower multipoles. This result can be interpreted in terms of additional contributions to the $23 \mathrm{GHz}$ Galactic non-thermal emission, which could be mainly due to anomalous dust.

A direct application of the presented analysis is to determine the level of contamination of the CMB anisotropies due to the Galactic diffuse synchrotron emission at different angular scales. The conclusions reported below refer to the cuts, which are more relevant for CMB measurements because of the large coverage. However, similar considerations could be repeated using the results of the local analysis, thus allowing the identification of the clearest sky areas, which is essential for the success of groundbased CMB experiments.

7. An important - although not unexpected - outcome of the cut analysis is that the amplitude of the APS for the northern hemisphere cuts above $\sim 20^{\circ}$ is raised by the presence of the NPS. Consequently, the results obtained separately for the two Galactic hemispheres were used to bracket the APS of the synchrotron emission.

8. We used the APS results at $408 \mathrm{MHz}$ and $1420 \mathrm{MHz}$ to determine the frequency spectral index to be adopted in the extrapolation to the microwave range for each coverage case. In particular, we found that $\beta_{(0.408-1.4) \mathrm{GHz}} \in[-3.2,-2.9]$ with an uncertainty of a few percent.

9. The extrapolation to $30 \mathrm{GHz}$ of the synchrotron APS obtained at $1420 \mathrm{MHz}$ led to a signal in good agreement with the upper limit fixed by COBE-DMR, thus supporting the reliability of our results. At this frequency the synchrotron emission constitutes a severe contamination of CMB anisotropies at the largest angular scales $(\ell \lesssim 40)$. Nevertheless, a cut at $\sim 20^{\circ}$ would reduce the synchrotron fluctuations to about half the cosmological ones. The same holds at $v \sim 70 \mathrm{GHz}$ for a cut at $\sim 5^{\circ}$ and the situation further improves when excluding a larger portion of the sky around the Galactic plane. This implies that even though the current treatment of the foregrounds does not permit an accurate removal of the synchrotron emission, the latter does not prevent the recovery of the bulk of the cosmological information encoded in the CMB temperature APS.

A deeper understanding of the foreground is indispensable to settle other important issues in the perspective of the forthcoming PLANCK mission. PLANCK should achieve a sensitivity comparable to the cosmic variance and its performance should be 
limited mainly by foregrounds, thanks to the extremely accurate control of all instrumental systematic effects (Burigana et al. 2004; Mennella et al. 2004).

A first issue is the estimate of the CMB temperaturepolarization APS, which carries information about the reionization history and the tensor-to-scalar ratio (see, e.g. Kogut 2003; Kogut et al. 2003, and references therein). Possible foreground residual contamination in the total intensity $\mathrm{CMB}$ anisotropy map would affect fine analysis based on the estimate of the crosscorrelation APS, also because the polarized component of the cosmological signal is orders of magnitude lower.

Another issue is the evaluation of the Gaussianity of the primordial fluctuations, which in the standard inflationary paradigm $^{8}$ generate the structures observed in the Universe today. Gaussianity tests are a powerful tool, complementary to the tests exploiting the APS, which allow us to probe the "concordance" model (Spergel et al. 2007) and also to distinguish among inflationary models (Bartolo et al. 2004). The level of non-Gaussianity predicted by the "concordance" model cannot be detected by WMAP, but in principle it should be observable with PLANCK. Galactic foregrounds are non-Gaussian and anisotropic and even low-level contamination in the maps can produce detectable non-Gaussianities (see, e.g. Naselsky et al. 2005), although they have minimal effects on the estimated APS (Hinshaw et al. 2003). Consequently, the foreground removal has to be extremely accurate, so as not to limit PLANCK in verifying this crucial $^{9}$ prediction.

Acknowledgements. We are grateful to R. Wielebinski for a careful reading of the original manuscript. We wish to thank G. De Zotti, L. Toffolatti and R. Rebolo for helpful discussions. L.L.P. warmly thanks R. Wielebinski and A. Zensus for granting a post-doc fellowship. C.B. acknowledge the support by the ASI contract "Planck LFI Activity of Phase E2". We are grateful to M. Genghini for technical support. Some of the results in this paper have been derived using the HEALPix (Górski et al. 2005) package. The availability of the WMAP 3 -yr maps is acknowledged. We warmly thank the anonymous referee for useful comments.

\section{References}

Alishahiha, M., Silverstein, E., \& Tong, D. 2004, Phys. Rev., 70, 12

Arfken, G. B., \& Weber, H. J., 2001, Mathematical Methods for Physicists (Harcourt Academy Press), 893

Arkani-Hamed, N., Creminelli, P., Mukohyama, S., \& Zaldarriaga, M. 2004, JCAP, 4, 1

Baccigalupi, C., Burigana, C., Perrotta, F., et al. 2001, A\&A, 372, 8

Banday, A. J., \& Wolfendale, A. W. 1990, MNRAS, 245, 182

Banday, A. J., \& Wolfendale, A. W. 1991, MNRAS, 252, 462

Bartolo, N., Komatsu, E., Matarrese, S., \& Riotto, A. 2004, Phys. Rev., 402, 103 Bennett, C. L., Hill, R. S., Hinshaw, G., et al. 2003, ApJS, 148, 97

Bouchet, F. R., \& Gispert, R. 1999, NewA, 4, 443

Bouchet, F. R., Prunet, S., \& Sethi, S. 1999, MNRAS, 302, 663

Burigana, C., Finelli, F., Salvaterra, R., et al. 2004, MmSAI, 5, 415

Burigana, C., La Porta, L., Reich, P., \& Reich, W. 2006, AN, 327, 491

Chepurnov, A. V. 1998, A\&AT, 17, 281

Chiang, L.-Y., Coles, P., \& Naselsky, P. D. 2007, JCAP, 1, 21
Cho, J., \& Lazarian, A. 2002, ApJ, 575, L63

Cho, J., \& Lazarian, A. 2003, NewAR, 47, 1143

Davies, R. D., Dickinson, C., Banday, A. J., et al. 2006, MNRAS, 370, 1125

De Oliveira-Costa, A, Tegmark, M., Davies, R. D., et al. 2004, ApJ, 606, L89

Dickinson, C., Davies, R. D., \& Davis, R. J. 2003, MNRAS, 341, 369

Draine, B. T., \& Lazarian, A. 1998, ApJ, 494, L19

Egger, R. J., \& Aschenbach, B. 1995, A\&A, 294, L25

Franceschini, A., Toffolatti, L., Danese, L., De Zotti, G. 1989, ApJ, 344, 35

Finkbeiner, D. P. 2003, ApJS, 146, 407

Finkbeiner, D. 2004, ApJ, 614, 186

Finkbeiner, D. P., Davis, M., \& Schlegel, D. J. 1999, ApJ, 524, 867

Giardino, G., Banday, A. J., Górski, K. M., et al. 2001, A\&A, 371, 708

Górski, K. M., Hivon, E., Banday, A. J., et al. 2005, ApJ, 622, 759

Haslam, C. G. T. 1974, A\&AS, 15, 333

Haslam, C. G. T., Salter, C. J., Stoffel, H., \& Wilson, W. E. 1982, A\&AS, 47, 1

Hildebrandt, S. R., Rebolo, R., Rubiño-Martín, J. A., et al. 2007, MNRAS, submitted [arXiv: astro-ph/07061873]

Hinshaw, G., Spergel, D.N., Verde, L., et al. 2003, ApJS, 148, 135

Hinshaw, G., Nolta, M. R., Bennett, C. L., et al. 2007, ApJS, 170, 288

James, F., \& Roos, M. 1975, Comput. Phys. Comm., 10, 343

Jamrozy, M. 2004, A\&A, 419, 63

Kogut, A. 2003, NewAR, 47, 977

Kogut, A., Banday, A. J., Bennett, C. L., et al. 1996, ApJ, 464, L5

Kogut, A., Spergel, D. N., Barnes, C., et al. 2003, ApJS, 148, 161

La Porta, L. 2007, Ph.D. Thesis, Bonn University, Germany

La Porta, L., Reich, P., Burigana, C., \& Reich, W. 2005, MPIfR-Memo, 1

Linde, A. 2005, NewAR, 49, 35

Lyth, D. H., \& Riotto, A. A. 1999, Phys. Rep., 314, 1

Lyth, D. H., Ungarelli, C., \& Wands, D. 2003, Phys. Rev., 67, 2

Mennella, A., Baccigalupi, C., Balbi, A., et al. 2004, Recent Research

Developments in Astronomy \& Astrophysics, 2

[arXiv: astro-ph/0402528]

Naselsky, P. D., \& Verkhodanov, O. V. 2006, MNRAS, submitted

[arXiv: astro-ph/0609409]

Naselsky, P. D., Chiang, L.-Y., Olesen, P., \& Novikov, I. D. 2005, Phys. Rev., 72,063512

Naselsky, P. D., Novikov, I. D., \& Chiang, L.-Y. 2006, ApJ, 642, 617

Negrello, M., González-Nuevo, J., Magliocchetti, M., et al. 2005, MNRAS, 358, 896

Negrello, M., Magliocchetti, M., \& De Zotti, G. 2006, MNRAS, 368, 935

Paladini, R., De Zotti, G., Davies, R. D., \& Giard, M. 2005, MNRAS, 360, 1545

Platania, P., Burigana, C., Maino, D., et al. 2003, A\&A, 410, 847

Prandoni, I., Gregorini, L., Parma, P., et al. 2001, A\&A, 365, 392

Reich, W. 1982, A\&AS, 48, 219

Reich, P., \& Reich, W. 1986, A\&AS, 63, 205

Reich, P., \& Reich, W. 1988, A\&AS, 74, 7

Reich, P., Testori, J. C., \& Reich, W. 2001, A\&A, 376, 861

Reich, P., Reich, W., \& Testori, J. C. 2004, Proceeding The Magnetized Interstellar Medium, Antalya, Turkey, ed. B. Uyaniker, W. Reich, \& R. Wielebinski, (Katlenburg-Lindau: Copernicus GmbH), 63

Rybicki, G. B., \& Lightman, A. P. 1979, Radiative processes in astrophysics (New York: Wiley-Interscience)

Salter, C. J. 1983, BASI, 11, 1

Spergel, D. N., Bean, R., Doré, O., et al. 2007, ApJS, 170, 377

Strong, A. W., Moskalenko, I. V., \& Ptuskin, V. S. 2007, Annu. Rev. Nucl. Part. Sci., 57, 285

Tauber, J. 2004, AdSpR, 34, 91

Tegmark, M., \& Efstathiou, G. 1996, MNRAS, 281, 1297

Tegmark, M., Eisenstein, D. J., Hu, W., \& De Oliveira-Costa, A. 2000, ApJ, 530, 133

Toffolatti, L., Argüeso Gómez, F., De Zotti, G., et al. 1998, MNRAS, 297, 117

Testori, J. C., Reich, P., Bava, J. A., et al. 2001, A\&A, 368, 1123

\footnotetext{
8 Simple (standard) inflationary scenarios (see the reviews by Lyth \& Riotto 1999; and Linde 2005) predict the existence of Gaussian density fluctuations and of gravitational waves with a nearly scale-invariant spectrum.

9 Standard cosmologies predict a minimum level of non-Gaussianity for the the primordial perturbations. In alternative cosmologies (Lyth et al. 2003; Arkani-Hamed et al. 2004; Alishahiha et al. 2004) such a lower limit is even higher. Consequently, detection or non-detection of non-Gaussianities sheds light on the physics of the early Universe.
} 